Journal of

Business \&

Economic

Statistics

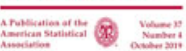

\title{
Including Covariates in the Regression Discontinuity Design
}

\section{Markus Frölich \& Martin Huber}

To cite this article: Markus Frölich \& Martin Huber (2019) Including Covariates in the Regression Discontinuity Design, Journal of Business \& Economic Statistics, 37:4, 736-748, DOI: 10.1080/07350015.2017.1421544

To link to this article: https://doi.org/10.1080/07350015.2017.1421544

\section{+ View supplementary material $\sqsubset$}

曲 Published online: 05 Sep 2018.

Submit your article to this journal $\pi$

III Article views: 1232

Q View related articles $ᄃ$

View Crossmark data ־

Citing articles: 5 View citing articles $\pi$ 


\title{
Including Covariates in the Regression Discontinuity Design
}

\author{
Markus FröLICH \\ Center for Evaluation and Development (C4ED), University of Mannheim, J-PAL, L7, 3-5, D-68131 Mannheim, \\ Germany (froelich@c4ed.org, froelich@uni-mannheim.de) \\ Martin HuBER \\ Department of Economics, University of Fribourg, Bd. de Pérolles 90, $\mathrm{CH}-1700$ Fribourg, Switzerland \\ (martin.huber@unifr.ch)
}

\begin{abstract}
This article proposes a fully nonparametric kernel method to account for observed covariates in regression discontinuity designs (RDD), which may increase precision of treatment effect estimation. It is shown that conditioning on covariates reduces the asymptotic variance and allows estimating the treatment effect at the rate of one-dimensional nonparametric regression, irrespective of the dimension of the continuously distributed elements in the conditioning set. Furthermore, the proposed method may decrease bias and restore identification by controlling for discontinuities in the covariate distribution at the discontinuity threshold, provided that all relevant discontinuously distributed variables are controlled for. To illustrate the estimation approach and its properties, we provide a simulation study and an empirical application to an Austrian labor market reform. Supplementary materials for this article are available online.
\end{abstract}

KEY WORDS: Causal effect; Complier; Endogeneity; LATE; Nonparametric regression; Treatment effect.

\section{INTRODUCTION}

The regression discontinuity design (RDD) has received tremendous attention in many fields, for example, labor markets, political economy, health, education, psychology, criminology, as a credible approach to identifying causal effects without having to resort to fully randomized experiments. Hahn, Todd, and van der Klaauw (2001) formalized the assumptions required to identify causal effects in the RDD and provided nonparametric (local linear) estimators. Porter (2003) complemented their work by alternative estimators. Lee and Card (2008) considered the case when the forcing variable is discrete. McCrary (2008) proposed a test for the manipulation of the running variable related to the continuity of its density function. Imbens and Lemieux (2008), van der Klaauw (2008), and Lee and Lemieux (2010) surveyed the applied and theoretical literature on the RDD. Imbens and Kalyanaraman (2012) discussed optimal bandwidth selection in terms of squared error loss, while Calonico, Cattaneo, and Titiunik (2014) proposed methods for robust inference along with optimal bandwidth selection. Dong (2014) presented an alternative to some of the identifying assumptions in Hahn, Todd, and van der Klaauw (2001).

In this article, the regression discontinuity approach is extended to incorporate covariates in a fully nonparametric way. Our estimator is based on a local nonparametric regression approach, that is, kernel-based estimation, which allows deriving closed-form expressions for bias and variance. (An alternative approach could use global nonparametric methods such as sieves or polynomials of increasing order. However, such global methods, which are capable of fitting regression curves at many points by means of extrapolation, may perform poorly in the RDD, where a good fit is only needed at the treatment threshold, see Gelman and Imbens (2018). Extrapolation from far-away data points is also inherent in linear regression where one linearly controls for covariates.) Consider the setup of the RDD: $D$ is a binary treatment indicator, $Y$ is the outcome variable of interest, and $Z$ is the "forcing variable" with a known threshold $z_{0}$ at which the treatment probability $\operatorname{Pr}(D=1 \mid Z)$ is discontinuous. There are various motivations for accounting for covariates, denoted by $X$. A first reason is variance reduction, which is well known for the parametric case. But gains in precision can also be achieved in the nonparametric setup, as flexibly including covariates and averaging them out in an appropriate way reduces the asymptotic variance of the estimated treatment effect. We show that under mild regularity conditions, incorporating covariates permits estimating the treatment effect at the rate for one-dimensional nonparametric regression, that is, $n^{-\frac{2}{5}}$ (where $n$ is the sample size), irrespective of the dimension of the continuously distributed elements in $X$. Hence, the curse of dimensionality does not apply due to smoothing over $X$.

Second, as pointed out by Imbens and Lemieux (2008), covariates may mitigate small sample biases in cases where the number of observations close to the threshold $z_{0}$ is small such that one has to include observations in the estimation that are further apart and may potentially differ in $X$. Controlling for $X$ might eliminate some of the bias that is introduced by

DOI: 10.1080/07350015.2017.1421544 
observations further away from the threshold, as illustrated by Black, Galdo, and Smith (2007). However, biases related to unobserved characteristics cannot be accounted for.

Third, we also permit for situations where the density $f(X \mid Z)$ is discontinuous at $z_{0}$, which may point to a failure of the RDD assumptions, see Lee (2008), such that the simple RDD estimator is generally inconsistent. Our approach nevertheless identifies a local treatment effect in cases in which $X$ contains all variables that (i) are imbalanced around the threshold and (ii) affect the outcome variable. With this respect, our contribution distinguishes itself from a more recent article on RDD with covariates by Calonico et al. (2018), who assume $f(X \mid Z)$ to be continuous at $z_{0}$. Under that stronger identifying condition not needed here, Calonico et al. (2018) discussed potential precision gains when linearly (rather than nonparametrically as in our method) controlling for $X$ and provided methods for optimal bandwidth selection and robust inference.

One example for $f(X \mid Z)$ being discontinuous at $z_{0}$ is "classical confounding" where manipulation of $Z$ at the threshold is selective with respect to characteristics that may also affect the outcome, see, for instance, Urquiola and Verhoogen (2009). If all confounding characteristics are observed in the data, our method yields the treatment effect on compliers at the threshold. See also van der Klaauw (2008) for confounding in the context of dynamic treatment assignment, where observed earlier treatment eligibility or participation $(X)$ jointly affects the (current) forcing variable $Z$ and $Y$. As a further example, consider the case when $Z$ not only affects $D$, but also further variables that affect $Y$. This may occur in spatial RDDs where $Z$ is based on distance to geographical borders. Eugster et al. (2017), for instance, use the (mainly French and German) language border within administrative units of Switzerland to estimate the effects of culture on unemployment. The authors consider a measure of the "taste for leisure" as one particular indicator of culture. However, in addition to this treatment variable, further community-based covariates that are likely affected by culture also change discontinuously at the border. Controlling for $X$ is therefore necessary as $Z$ would otherwise violate the exclusion restriction with respect to $Y$ at the threshold through its influence on $X$. Identification of a causal effect is, however, only obtained if $X$ are not "bad controls" which are affected by unobservables that also influence $Y$.

The remainder of this article is organized as follows. Section 2 discusses the identification of the treatment effect in the presence of covariates. Section 3 proposes two estimators and examines their properties and shows that one of them achieves the $n^{-\frac{2}{5}}$ convergence rate. Section 4 provides a simulation study that (among others) illustrates the implications of confounding related to observed covariates at the threshold when applying RDD with and without controlling for $X$. Section 5 presents an empirical application to Austrian labor market reform previously considered by Lalive (2008) to estimate the effect of age-dependent eligibility to unemployment benefits on unemployment duration. As employees at risk of becoming unemployed might negotiate the exact date of dismissal with their employers, manipulation at the age threshold is a concern. We therefore control for a range of labor market-relevant characteristics that are potential confounders and find our results to differ from RDD without $X$. Section 6 concludes.

\section{RDD WITH COVARIATES}

We define causal effects using the potential-outcome notation in the framework known as the Neyman-Fisher-Rubin causal model. (See Neyman (1923), Fisher (1935), and Rubin (1978).) Following the setup of Hahn, Todd, and van der Klaauw (2001), let $D_{i} \in\{0,1\}$ be a binary treatment variable, let $Y_{i}^{0}, Y_{i}^{1}$ be the individual potential outcomes, and $Y_{i}^{1}-Y_{i}^{0}$ the individual treatment effect. The potential outcomes as well as the treatment effects $Y_{i}^{1}-Y_{i}^{0}$ are permitted to vary across individuals, that is, no constant treatment effect is assumed. Let $Z_{i}$ be a variable that influences the treatment variable in a discontinuous way.

In the literature, two distinct designs are examined: the sharp design where $D_{i}$ changes for everyone at a known threshold $z_{0}$, and the fuzzy design where $D_{i}$ changes only for a subset of individuals. In the sharp design (Trochim 1984), participation status is given by a deterministic function of $Z$, for example,

$$
D_{i}=1\left(Z_{i} \geq z_{0}\right) .
$$

This implies that all individuals change program participation status exactly at $z_{0}$. The fuzzy design, on the other hand, permits $D$ to also depend on other factors but assumes that the treatment probability changes discontinuously at $z_{0}$ :

$$
\lim _{\varepsilon \rightarrow 0} E\left[D \mid Z=z_{0}+\varepsilon\right]-\lim _{\varepsilon \rightarrow 0} E\left[D \mid Z=z_{0}-\varepsilon\right] \neq 0 .
$$

Note that the fuzzy design includes the sharp design as a special case when the left-hand side of (2) is equal to one. For this reason, the subsequent discussion mostly focuses on the more general fuzzy design. (Battistin and Rettore (2008) introduced the mixed sharp fuzzy design as a special case of the fuzzy design.) See Hahn, Todd, and van der Klaauw (2001) for more details.

Identification is feasible under the continuity of the mean potential outcomes at $z_{0}$ and relies on comparing the observed outcomes of those individuals to the left of the threshold with those to the right. In addition to continuity of $E\left[Y^{d} \mid Z=z\right]$ in $z$ at $z_{0}$ for $d=\{0,1\}$, Hahn, Todd, and van der Klaauw (2001) considered two alternative identifying assumptions:

$$
\text { HTK1: } \quad Y_{i}^{1}-Y_{i}^{0} \Perp D_{i} \mid Z_{i} \quad \text { for } Z_{i} \text { near } z_{0}
$$

or

HTK2: $\left\{Y_{i}^{1}-Y_{i}^{0}, D_{i}(z)\right\} \Perp Z_{i} \quad$ near $z_{0} \quad$ and there exists $e>0$

$$
\text { such that } D_{i}\left(z_{0}+\varepsilon\right) \geq D_{i}\left(z_{0}-\varepsilon\right) \text { for all } 0<\varepsilon<e \text {. }
$$

Assumption (3) is a local selection on observables assumption and identifies the average treatment effect at the threshold: $E\left[Y^{1}-Y^{0} \mid Z=z_{0}\right]$. Assumption (4) is an instrumental variables assumption that identifies a local average treatment effect (LATE) for a local group of compliers at the threshold:

$$
\lim _{\varepsilon \rightarrow 0} E\left[Y^{1}-Y^{0} \mid D\left(z_{0}+\varepsilon\right)>D\left(z_{0}-\varepsilon\right), Z=z_{0}\right] .
$$

In the sharp design, everyone is a complier at $z_{0}$ and assumption (3) is meaningless (i.e., has no identifying power) such that one needs assumption (4). In the fuzzy design, one typically invokes (4), since the conditional independence assumption (3) does not permit treatment selection based on individual gains $Y_{i}^{1}-Y_{i}^{0}$. It is worth mentioning that Dong (2014) recently showed that alternatively to (4), identification of the LATE is obtained by making a continuity assumption of $Z$ in the neighborhood of 
$z_{0}$. (Continuity of $Z$ implies the smoothness of mean potential outcomes conditional on compliance behavior and of the shares of subgroups defined upon compliance at the threshold, which is sufficient for identification.)

In the following, we introduce observed covariates $X_{i}$ and assume that (4) is valid conditional on $X$. As an example, suppose that there exists a liberalized education market in which schools may charge tuition fees, and that by law classes must be split if the number of students surpasses a particular threshold. As argued in Urquiola and Verhoogen (2009) for the case of Chile, schools close to the threshold might adjust tuition fees, thereby causing discontinuities in the admitted students' socioeconomic characteristics such as household income and parents' education. Assume that the latter variables also affect the outcome of interest, for example, students' educational degree, which implies a violation of HTK2 when assessing the educational effect of class size. However, if household income, parents' education, and all other variables imbalanced at the threshold and affecting the outcome are observed, (4) holds conditional on $X_{i}$. (Whether it is plausible to assume that all imbalanced covariates affecting the outcome are observed depends on the empirical problem and the richness of data. In the context of Urquiola and Verhoogen (2009), for instance, ambition might (in addition to parents' education and household income) play a role for selectively (re)placing students into particular class sizes. One would therefore want to condition on a rich set of socio-economic household characteristics and personality traits, for example, provided by means of a household survey.) By an analogous reasoning as in HTK, and further assumptions made precise below, it follows immediately that the treatment effect on the local compliers conditional on $X$ is identified as

$$
\begin{aligned}
& \lim _{\varepsilon \rightarrow 0} E\left[Y^{1}-Y^{0} \mid X, D\left(z_{0}+\varepsilon\right)>D\left(z_{0}-\varepsilon\right), Z=z_{0}\right] \\
& \quad=\frac{m^{+}\left(X, z_{0}\right)-m^{-}\left(X, z_{0}\right)}{d^{+}\left(X, z_{0}\right)-d^{-}\left(X, z_{0}\right)},
\end{aligned}
$$

where $m^{+}(X, z)=\lim _{\varepsilon \rightarrow 0} E[Y \mid X, Z=z+\varepsilon]$ and $m^{-}(X, z)=$ $\lim _{\varepsilon \rightarrow 0} E[Y \mid X, Z=z-\varepsilon]$ and $d^{+}(X, z)$ and $d^{-}(X, z)$ defined analogously with $D$ replacing $Y$.

In this article, however, we focus on identifying and estimating the unconditional effect

$$
\lim _{\varepsilon \rightarrow 0} E\left[Y^{1}-Y^{0} \mid D\left(z_{0}+\varepsilon\right)>D\left(z_{0}-\varepsilon\right), Z=z_{0}\right],
$$

that is, the effect on all compliers without conditioning on $X$. We identify this effect by first controlling for $X$ and thereafter averaging over $X$. There are at least three reasons, why estimating the unconditional effect (6) is interesting (or even more interesting than the conditional effect (5)). First, for the purpose of evidence-based policy-making a small number of summary measures can be more easily conveyed to policy makers and the public than a large number of estimated effects at every value of $X$. Second, unconditional effects can be estimated more precisely than conditional effects. Third, the definition of unconditional effects does not depend on the variables included in $X$. (This, of course, is only true if $X$ exclusively contains pretreatment variables.) One can therefore consider different sets of control variables $X$ and still estimate the same object, which is useful for examining robustness of the results to the set of control variables. See also Frölich (2007).
For showing identification of the unconditional effect (6), we first introduce some further notation. Let $\mathcal{N}_{\varepsilon}$ be a symmetric $\varepsilon$ neighborhood about $z_{0}$ and partition $\mathcal{N}_{\varepsilon}$ into $\mathcal{N}_{\varepsilon}^{+}=\{z: z \geq$ $\left.z_{0}, z \in \mathcal{N}_{\varepsilon}\right\}$ and $\mathcal{N}_{\varepsilon}^{-}=\left\{z: z<z_{0}, z \in \mathcal{N}_{\varepsilon}\right\}$. According to their reaction to the instrument $z$ over $\mathcal{N}_{\varepsilon}$ we can partition the population into four subpopulations:

$$
\begin{array}{llllllll}
\tau_{i, \varepsilon}=a & \text { if } & D_{i}(z)=1 & \forall z \in \mathcal{N}_{\varepsilon}^{-} & \text {and } & D_{i}(z)=1 & \forall z \in \mathcal{N}_{\varepsilon}^{+} \\
\tau_{i, \varepsilon}=n & \text { if } & D_{i}(z)=0 & \forall z \in \mathcal{N}_{\varepsilon}^{-} & \text {and } & D_{i}(z)=0 & \forall z \in \mathcal{N}_{\varepsilon}^{+} \\
\tau_{i, \varepsilon}=c & \text { if } & D_{i}(z)=0 & \forall z \in \mathcal{N}_{\varepsilon}^{-} & \text {and } & D_{i}(z)=1 & \forall z \in \mathcal{N}_{\varepsilon}^{+} \\
\tau_{i, \varepsilon}=d & \text { if } & D_{i}(z)=1 & \forall z \in \mathcal{N}_{\varepsilon}^{-} & \text {and } & D_{i}(z)=0 & \forall z \in \mathcal{N}_{\varepsilon}^{+} .
\end{array}
$$

These subpopulations are a straightforward extension of the LATE concept of Imbens and Angrist (1994). The first group contains those units that will always be treated (if $Z \in \mathcal{N}_{\varepsilon}$ ), the second contains those that will never be treated (if $Z \in \mathcal{N}_{\varepsilon}$ ), and the third and fourth group contains the units that are treated only on one side of $z_{0}$. (In the appendix, we also consider a possible fifth group of indefinite units, for which no left-limit of $D_{i}(z)$ may exist. We assume this group to not exist, that is, we require that all units have well-defined left-limits of $D_{i}(z)$.) We will assume that the fourth group, that is, the "defiers," has measure zero for $\varepsilon$ sufficiently small. Note that in the sharp design, everyone is a complier for any $\varepsilon>0$.

Under the following assumption, we can identify the treatment effect for the local compliers, that is, for those who switch from $D=0$ to 1 at $z_{0}$. (The conditions in Assumption 1 are very similar, but a little weaker, to a conditional-on- $X$ version of (4).) It is assumed throughout that the covariates $X$ are continuously distributed with a Lebesgue density. This assumption is made for convenience to ease exposition, particularly in the derivation of the asymptotic distributions later on. Discrete covariates can (at the expense of more cumbersome notation) easily be included in $X$, as the derivation of the asymptotic distribution only depends on the number of continuous regressors in $X$, while discrete variables do not affect the asymptotic properties. In fact, identification does not require any continuous $X$ variables. Only $Z$ has to be continuous near $z_{0}$, but could have masspoints elsewhere.

Assumption 1. For a symmetric neighborhood $\mathcal{N}_{\varepsilon}$ about $z_{0}$ and for almost every $X$

(i) Existence of compliers

$$
\lim _{\varepsilon \rightarrow 0} \operatorname{Pr}\left(\tau_{\varepsilon}=c \mid Z \in \mathcal{N}_{\varepsilon}\right)>0
$$

(ii) Monotonicity $\lim _{\varepsilon \rightarrow 0} \operatorname{Pr}\left(\tau_{\varepsilon}=c \mid Z \in \mathcal{N}_{\varepsilon}\right)+\operatorname{Pr}\left(\tau_{\varepsilon}=a \mid Z \in \mathcal{N}_{\varepsilon}\right)+$ $\operatorname{Pr}\left(\tau_{\varepsilon}=n \mid Z \in \mathcal{N}_{\varepsilon}\right)=1$

(iii) Independent IV $\lim _{\varepsilon \rightarrow 0} \operatorname{Pr}\left(\tau_{\varepsilon}=t \mid X, Z \in \mathcal{N}_{\varepsilon}^{+}\right)-\operatorname{Pr}\left(\tau_{\varepsilon}=t \mid X, Z \in\right.$ $\left.\mathcal{N}_{\varepsilon}^{-}\right)=0$ for $t \in\{a, n, c\}$

(iv) IV Exclusion

$$
\begin{aligned}
& \lim _{\varepsilon \rightarrow 0} E\left[Y^{1} \mid X, Z \in \mathcal{N}_{\varepsilon}^{+}, \tau_{\varepsilon}=t\right]-E\left[Y^{1} \mid X, Z \in \mathcal{N}_{\varepsilon}^{-},\right. \\
& \left.\tau_{\varepsilon}=t\right]=0 \text { for } t \in\{a, c\} \\
& \lim _{\varepsilon \rightarrow 0} E\left[Y^{0} \mid X, Z \in \mathcal{N}_{\varepsilon}^{+},\right. \\
& \left.\tau_{\varepsilon}=t\right]-E\left[Y^{0} \mid X, Z \in \mathcal{N}_{\varepsilon}^{-}, \tau_{\varepsilon}=t\right]=0 \text { for } t \in\{n, c\}
\end{aligned}
$$

(v) Common support

$$
\lim _{\varepsilon \rightarrow 0} \operatorname{Supp}\left(X \mid Z \in \mathcal{N}_{\varepsilon}^{+}\right)=\lim _{\varepsilon \rightarrow 0} \operatorname{Supp}\left(X \mid Z \in \mathcal{N}_{\varepsilon}^{-}\right)
$$

(vi) Density at threshold

$F_{Z}(z)$ is differentiable at $z_{0}$ and $f_{Z}\left(z_{0}\right)>0$ 
$\lim _{\varepsilon \rightarrow 0} F_{X \mid Z \in \mathcal{N}_{\varepsilon}^{+}}(x)$ and $\lim _{\varepsilon \rightarrow 0} F_{X \mid Z \in \mathcal{N}_{\varepsilon}^{-}}(x)$ exist and are differentiable in $x$ with pdf $f^{+}\left(x \mid z_{0}\right)$ and $f^{-}\left(x \mid z_{0}\right)$, respectively.

(vii) Bounded moments

$E\left[Y^{1} \mid X, Z\right]$ and $E\left[Y^{0} \mid X, Z\right]$ are bounded away from \pm infinity a.s. over $\mathcal{N}_{\varepsilon}$

Concerning notation, $f^{+}\left(x, z_{0}\right)=f^{+}\left(x \mid z_{0}\right) f\left(z_{0}\right)$ refers to the joint density of $X$ and $Z$ whereas $f^{+}\left(x \mid z_{0}\right)$ refers to the conditional density of $X$.

This assumption requires that in a neighborhood about $z_{0}$, the threshold acts like a local instrumental variable. Assumptions 1(i) to (iv) are instrumental variable assumptions for a binary instrument, as discussed, for example, in Imbens (2001). The monotonicity assumption 1(ii) rules out defiers at the threshold $z_{0}$, while 1 (i) requires the existence of compliers. We note that 1(i) and 1(ii) could be relaxed to a local version of the compliers-defiers assumption of de Chaisemartin (2017), which allows for defiers under particular conditions, at the cost of identifying the effects only for a subset of compliers (the so-called "comvivors"). Assumptions 1(iii) and 1(iv) represent the exclusion restriction, conditional on $X$. Assumption 1(v) requires common support because we need to integrate over the support of $X$ in (7). (If this assumption is not satisfied, one can redefine (7) by restricting it to the common support.) Assumption 1(vi) implies positive density at $z_{0}$, such that observations close to $z_{0}$ exist.

We also assume the existence of the limit density functions $f^{+}\left(x \mid z_{0}\right)$ and $f^{-}\left(x \mid z_{0}\right)$ at the threshold $z_{0}$. So far, we do not assume anything about their continuity with respect to $z$. In other words, the conditional density could be discontinuous, that is, $f^{+}\left(x \mid z_{0}\right) \neq f^{-}\left(x \mid z_{0}\right)$, in which case controlling for $X$ is important for identification and thus consistent estimation, or it could be continuous, that is, $f^{+}\left(x \mid z_{0}\right)=f^{-}\left(x \mid z_{0}\right)$, in which case identification does not hinge on controlling for observed covariates. The latter may, however, reduce the variance of the point estimator, as discussed below. (Note that Assumption 1 is somewhat stronger than needed for identification. Assumptions (1i) to (1iv) could be replaced with other assumptions that identify the local treatment effect conditional on $X$. For instance, if local compliers and local defiers had the same treatment effect, one could drop the monotonicity assumption. In addition, the existence of a density function for $X$ is not needed.)

Assumption (1vii) requires the conditional expectation functions to be bounded from above and below in a neighborhood of $z_{0}$. It is invoked to permit interchanging the operations of integration and taking limits via the dominated convergence theorem. (This assumption is certainly stronger than needed and could be replaced with some other smoothness conditions on $E\left[Y^{d} \mid X, Z\right]$ in a neighborhood of $z_{0}$.)

Theorem 1 (Identification of complier treatment effect). Under Assumption 1, the local average treatment effect $\gamma$ for the subpopulation of local compliers is nonparametrically identified as

$$
\begin{aligned}
\gamma & =\lim _{\varepsilon \rightarrow 0} E\left[Y^{1}-Y^{0} \mid Z \in \mathcal{N}_{\varepsilon}, \tau_{\varepsilon}=c\right] \\
& =\frac{\int\left(m^{+}\left(x, z_{0}\right)-m^{-}\left(x, z_{0}\right)\right) \cdot \frac{f^{+}\left(x \mid z_{0}\right)+f^{-}\left(x \mid z_{0}\right)}{2} d x}{\int\left(d^{+}\left(x, z_{0}\right)-d^{-}\left(x, z_{0}\right)\right) \cdot \frac{f^{+}\left(x \mid z_{0}\right)+f^{-}\left(x \mid z_{0}\right)}{2} d x} .
\end{aligned}
$$

Proof. See the appendix.

Under Assumption 1, the treatment effect for the local compliers is identified as a ratio of two integrals, as shown in Theorem 1. The numerator in (7) is the intention-to-treat (ITT) effect of $Z$ on $Y$, weighted by the conditional density of $X$, at $z_{0}$. (In the limit, the density of $X$ conditional on $Z$ being within a symmetric neighborhood around $z_{0}$ is given by $\frac{f^{+}\left(x \mid z_{0}\right)+f^{-}\left(x \mid z_{0}\right)}{2}$.) The denominator in (7) gives the effect of $Z$ on $D$, that is, the fraction of compliers, at $z_{0}$. Thus, the ratio of integrals gives the ITT effect multiplied with the inverse of the number of compliers, corresponding to the LATE at $z_{0}$.

The ratio of integrals expression in (7) is obtained by applying iterated expectations to

$$
E\left[Y^{1}-Y^{0} \mid Z \in \mathcal{N}_{\varepsilon}, \tau_{\varepsilon}=c\right]
$$

to obtain

$$
=\int E\left[Y^{1}-Y^{0} \mid X=x, Z \in \mathcal{N}_{\varepsilon}, \tau_{\varepsilon}=c\right] \cdot f_{X \mid Z \in \mathcal{N}_{\varepsilon}, \tau_{\varepsilon}=c}(x) d x .
$$

Clearly, the density $f\left(X \mid Z \in \mathcal{N}_{\varepsilon}, \tau_{\varepsilon}=c\right)$ among the local compliers is not identified since the type $\tau_{\varepsilon}$ is unobservable. However, by applying Bayes' theorem to $f\left(X \mid Z \in \mathcal{N}_{\varepsilon}, \tau_{\varepsilon}=c\right)$ and replacing the first term in (8) with (5) (before taking limits), several terms cancel out and we obtain after various calculations the expression (7), which relies on observed variables only. See the supplementary appendix for detailed derivations. We thereby have identified the average effect. Similarly, we could identify quantile treatment effects by combining the previous derivations with the reasoning in Frölich and Melly (2013) and Frandsen, Frölich, and Melly (2012).

So far, we have identified the treatment effect for the compliers in the fuzzy design. Without restrictions on treatment effect heterogeneity, it is impossible to identify the effects for alwaysand never-participants since they would never change treatment status in a neighborhood of $z_{0}$. However, in the sharp design, everyone is a complier at $z_{0}$, that is, $d^{+}\left(x, z_{0}\right)-d^{-}\left(x, z_{0}\right)=1$, and the expression (7) simplifies to

$$
\begin{aligned}
\lim _{\varepsilon \rightarrow 0} E\left[Y^{1}-Y^{0} \mid Z \in \mathcal{N}_{\varepsilon}\right]= & \int\left(m^{+}\left(x, z_{0}\right)-m^{-}\left(x, z_{0}\right)\right) \\
& \cdot \frac{f^{+}\left(x \mid z_{0}\right)+f^{-}\left(x \mid z_{0}\right)}{2} d x .
\end{aligned}
$$

The estimand (9) in the sharp design is identical to the numerator of (7). The following discussion focusses on the estimation of (7), where the numerator and denominator of (7) are analyzed separately. Therefore, the asymptotic distribution of (9) in the sharp design is immediately obtained by using the results for the numerator of (7) only. We also note that the estimands (7) and (9) bear some resemblance to the partial means estimator of Newey (1994). Both the numerator and denominator of (7) have a partial means form, in that averages over the covariates $X$ are taken, at the left and the right limit at $z_{0}$.

Instead of generalizing assumption (4) to permit for further covariates $X$, we could alternatively start from the conditional independence assumption (3). To conserve space, we, however, do not analyze this in much detail since most applied work either uses a sharp design (where (3) is meaningless) or otherwise refers to (4). Consider an extension of (3) by including 
covariates $X$ :

$$
Y_{i}^{1}-Y_{i}^{0} \Perp D_{i} \mid X_{i}, Z_{i} \quad \text { for } Z_{i} \text { near } z_{0} .
$$

Analogously to the derivations in Hahn, Todd, and van der Klaauw (2001) it follows that

$$
E\left[Y^{1}-Y^{0} \mid X, Z=z_{0}\right]=\frac{m^{+}\left(X, z_{0}\right)-m^{-}\left(X, z_{0}\right)}{d^{+}\left(X, z_{0}\right)-d^{-}\left(X, z_{0}\right)} .
$$

Similarly to the derivations for Theorem 1 , one can show that the unconditional treatment effect for the population near the threshold is

$$
\begin{aligned}
E\left[Y^{1}-Y^{0} \mid Z=z_{0}\right]= & \int \frac{m^{+}\left(x, z_{0}\right)-m^{-}\left(x, z_{0}\right)}{d^{+}\left(x, z_{0}\right)-d^{-}\left(x, z_{0}\right)} \\
& \cdot \frac{f^{+}\left(x \mid z_{0}\right)+f^{-}\left(x \mid z_{0}\right)}{2} d x
\end{aligned}
$$

This expression differs from (7) and (9) in that it is an integral of a ratio and not a ratio of integrals. The results derived in Section 3 therefore do not apply to (11). In addition, expression (11) may be difficult to estimate in small samples as the denominator can be close to zero for some values of $x$. (This problem is of much less concern for estimators of (7) and (9) as those are based on a ratio of two integrals and not on an integral of a ratio. For those estimators the problem of very small denominators for some values of $X$ averages out.)

Instead of using (10), one might be willing to strengthen the latter assumption to

$$
Y_{i}^{1}, Y_{i}^{0} \Perp D_{i} \mid X_{i}, Z_{i} \quad \text { for } Z_{i} \text { near } z_{0} .
$$

This permits identifying the treatment effect as

$$
\begin{aligned}
E & {\left[Y^{1}-Y^{0} \mid Z=z_{0}\right] } \\
= & \int\left(E\left[Y \mid D=1, X=x, Z=z_{0}\right]-E\left[Y \mid D=0, X=x, Z=z_{0}\right]\right) \\
& \quad \frac{f^{+}\left(x \mid z_{0}\right)+f^{-}\left(x \mid z_{0}\right)}{2} d x,
\end{aligned}
$$

where $E\left[Y \mid D, X, Z=z_{0}\right]$ can be estimated by a combination of the left- and right-hand side limits. This approach does not exclusively rely on comparing observations across the threshold but also uses variation within either side of the threshold. The estimand has a similar structure as (7) and (9) and the estimation properties derived later could easily be extended to this case.

\section{ESTIMATION}

A straightforward estimator of (7) is

$$
\hat{\gamma}=\frac{\sum_{i=1}^{n}\left(\hat{m}^{+}\left(X_{i}, z_{0}\right)-\hat{m}^{-}\left(X_{i}, z_{0}\right)\right) \cdot K_{h}\left(\frac{Z_{i}-z_{0}}{h}\right)}{\sum_{i=1}^{n}\left(\hat{d}^{+}\left(X_{i}, z_{0}\right)-\hat{d}^{-}\left(X_{i}, z_{0}\right)\right) \cdot K_{h}\left(\frac{Z_{i}-z_{0}}{h}\right)},
$$

where $\hat{m}$ and $\hat{d}$ are nonparametric estimators and $K_{h}(u)$ is a kernel function. For the sharp design (9) the estimator simplifies to

$$
\frac{\sum\left(\hat{m}^{+}\left(X_{i}, z_{0}\right)-\hat{m}^{-}\left(X_{i}, z_{0}\right)\right) \cdot K_{h}\left(\frac{Z_{i}-z_{0}}{h}\right)}{\sum K_{h}\left(\frac{Z_{i}-z_{0}}{h}\right)} .
$$

For practical convenience, we will mostly work with product kernel functions below. Product kernel functions also have the advantage that one can easily incorporate discrete $X$ in the spirit of Racine and Li (2004). Define $\kappa$ and $\bar{\kappa}$ as univariate kernel functions, where $\kappa$ is a second-order kernel (assumed to be symmetric and integrating to one) and $\bar{\kappa}$ is a kernel of order $\lambda \geq 2$. The following kernel constants for $\kappa$ will be used later: $\bar{\mu}_{l}=\int_{-\infty}^{\infty} u^{l} \kappa(u) d u$ and $\bar{\mu}_{l}=\int_{0}^{\infty} u^{l} \kappa(u) d u$ and $\tilde{\mu}=$ $\frac{\bar{\mu}_{2}}{2}-\bar{\mu}_{1}^{2}$. (With symmetric kernel $\bar{\mu}_{0}=\frac{1}{2}$.) Furthermore define $\ddot{\mu}_{l}=\int_{0}^{\infty} u^{l} \kappa^{2}(u) d u$. (For the Epanechnikov kernel with support $[-1,1]$, that is, $K(u)=\frac{3}{4}\left(1-u^{2}\right) 1(|u|<1)$ the kernel constants are $\mu_{0}=1, \mu_{1}=\mu_{3}=\mu_{5}=0, \mu_{2}=0.2, \mu_{4}=6 / 70$, $\bar{\mu}_{0}=0.5, \bar{\mu}_{1}=3 / 16, \bar{\mu}_{2}=0.1, \bar{\mu}_{3}=1 / 16, \bar{\mu}_{4}=3 / 70$. The kernel constants for $\bar{\kappa}$ are defined as $\eta_{l}=\int_{-\infty}^{\infty} u^{l} \bar{\kappa}(u) d u$ and $\dot{\eta}_{l}=\int_{-\infty}^{\infty} u^{l} \bar{\kappa}^{2}(u) d u$. The kernel function $\bar{\kappa}$ being of order $\lambda$ means that $\eta_{0}=1$ and $\eta_{l}=0$ for $0<l<\lambda$ and $\eta_{\lambda} \neq 0$.)

We will consider two different choices for $K_{h}(u)$ in (13). The conventional choice would be to use a positive (i.e., second order) and symmetric kernel

$$
K_{h}(u)=\frac{1}{h} \kappa(u) .
$$

However, as shown below, the use of this "naive" kernel function (14) leads at best to a convergence rate of $n^{-\frac{1}{3}}$ of (13).

As an alternative, we consider a boundary kernel

$$
K_{h}(u)=\left(\bar{\mu}_{2}-\bar{\mu}_{1}|u|\right) \cdot \frac{1}{h} \kappa(u)
$$

in (13), and we will see that this leads to a convergence rate of $n^{-\frac{2}{5}}$ of (13), that is, the rate of univariate nonparametric regression. This is achieved through smoothing with implicit double boundary correction. (See, for example, Jones (1993) or Jones and Foster (1996) for similar boundary kernels, or Gasser and Müller (1979), Gasser, Müller, and Mammitzsch (1985), Müller (1991) or Tenreiro (2013) for a more general discussion on various forms of boundary kernels or boundary corrections including the derivation of optimal boundary kernels for density estimation, estimation of distribution functions, or estimation of nonparametric curves, etc.)

In the following, we will refer to estimator (13) with kernel

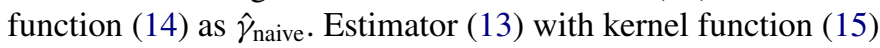
is denoted as $\hat{\gamma}_{\mathrm{RDD}}$. Because of the asymptotic properties derived below we recommend the use of $\hat{\gamma}_{\text {RDD }}$.

In either case, estimation proceeds in two steps and requires nonparametric first step estimates of $m^{+}, m^{-}, d^{+}$, and $d^{-}$. (In the sharp design (9), $d^{+}$and $d^{-}$are not estimated but set to 1 and 0 , respectively.) These can be estimated nonparametrically by considering only observations to the right or the left of $z_{0}$, respectively. Since this corresponds to estimation at a boundary point, local linear regression is suggested, which is known to display better boundary behavior than conventional NadarayaWatson kernel regression. $m^{+}\left(x, z_{0}\right)$ is estimated by local linear regression as the value of $a$ that solves

$$
\underset{a, b, c}{\arg \min } \sum_{j=1}^{n}\left(Y_{j}-a-b\left(Z_{j}-z_{0}\right)-c^{\prime}\left(X_{j}-x\right)\right)^{2} \cdot K_{j} I_{j}^{+},
$$

where $I_{j}^{+}=1\left(Z_{j}>z_{0}\right)$ and a product kernel is used

$$
K_{j}=K_{j}\left(x, z_{0}\right)=\kappa\left(\frac{Z_{j}-z_{0}}{h_{z}}\right) \cdot \prod_{l=1}^{L} \bar{\kappa}\left(\frac{X_{j l}-x_{l}}{h_{x}}\right),
$$


where $L$ is the dimension of $X$, and $\kappa$ and $\bar{\kappa}$ are univariate kernel functions with $\kappa$ a second-order kernel and $\bar{\kappa}$ a kernel of order $\lambda \geq 2$.

A result derived later will require higher-order kernels (i.e., $\lambda>2$ ) if the number of continuous regressors is larger than 3 . For applications with at most three continuous regressors, a second-order kernel will suffice such that $\bar{\kappa}=\kappa$ can be chosen. Note that three different bandwidths $h_{z}, h_{x}, h$ are used. $h$ is the bandwidth in the matching estimator (13) to compare observations to the left and right of the threshold, whereas $h_{z}$ and $h_{x}$ determine the local smoothing area for the local linear regression in (16), which uses observations only to the right or only to the left of the threshold. We need some smoothness assumptions as well as conditions on the bandwidth values. (Note that the above setup includes global linear regression for the special case where all bandwidth values are set to infinity. In this case, the estimator (16) corresponds to a linear regression using only data points to the right; and analogously on the left-hand side. While a bandwidth value of infinity minimizes variance it could lead to a large bias if the true regression curve is nonlinear. The estimator analyzed below seeks to minimize mean squared error, that is, the sum of the squared bias and variance.)

\section{Assumption 2.}

(i) IID sampling: The data $\left\{\left(Y_{i}, D_{i}, Z_{i}, X_{i}\right)\right\}$ are iid from $\mathbb{R} \times$ $\mathbb{R} \times \mathbb{R} \times \mathbb{R}^{L}$

(ii) Smoothness:

- $m^{+}(x, z), m^{-}(x, z), d^{+}(x, z), d^{-}(x, z)$ are $\lambda$ times continuously differentiable with respect to $x$ at $z_{0}$ with $\lambda$ th derivative Hölder continuous in an interval around $z_{0}$,

- $f^{+}(x, z)$ and $f^{-}(x, z)$ are $\lambda-1$ times continuously differentiable with respect to $x$ at $z_{0}$ with $(\lambda-1)$ th derivative Hölder continuous in an interval around $z_{0}$,

- $m^{+}(x, z), d^{+}(x, z)$, and $f^{+}(x, z)$ have two continuous right derivatives with respect to $z$ at $z_{0}$ with second derivative Hölder continuous in an interval around $z_{0}$,

- $m^{-}(x, z), d^{-}(x, z)$, and $f^{-}(x, z)$ have two continuous left derivatives with respect to $z$ at $z_{0}$ with second derivative Hölder continuous in an interval around $z_{0}$,

(iii) the univariate Kernel functions $\kappa$ and $\bar{\kappa}$ in (17) are symmetric, bounded, Lipschitz, integrate to one and are zero outside a bounded set; $\kappa$ is a second-order kernel and $\bar{\kappa}$ is a kernel of order $\lambda$,

(iv) Bandwidths: The bandwidths satisfy $h, h_{z}, h_{x} \rightarrow 0$ and $n h \rightarrow \infty$ and $n h_{z} \rightarrow \infty$ and $n h_{z} h_{x}^{L} \rightarrow \infty$.

(v) Conditional variances: The left and right limits of the conditional variances $\lim _{\varepsilon \rightarrow 0} E\left[\left(Y-m^{+}(X, Z)\right)^{2} \mid X, Z=\right.$ $z+\varepsilon]$ and $\lim _{\varepsilon \rightarrow 0} E\left[\left(Y-m^{-}(X, Z)\right)^{2} \mid X, Z=z-\varepsilon\right]$ exist at $z_{0}$.

\subsection{Properties of $\hat{\gamma}_{\text {naive }}$}

With these preliminaries, we consider the properties of $\hat{\gamma}_{\text {naive }}$ and $\hat{\gamma}_{\mathrm{RDD}}$. The estimator $\hat{\gamma}_{\text {naive }}$ is, in essence, a combination between local linear regression in the first step and Nadaraya-Watson regression in the second step. Although this estimator appears to be the most obvious one for estimating (7), it has worse statistical properties than $\hat{\gamma}_{\mathrm{RDD}}$ in the sense that it achieves a lower rate of convergence. This is due to the missing boundary correction in the second step.
Proposition 1 (Asymptotic properties of $\hat{\gamma}_{\text {naive }}$ ). Under Assumptions 1, 2, and 3, the bias and variance terms of $\hat{\gamma}_{\text {naive }}$, which is the estimator (13) with kernel function (14), are of order

$$
\begin{aligned}
\operatorname{Bias}\left(\hat{\gamma}_{\text {naive }}\right) & =O\left(h+h_{z}^{2}+h_{x}^{\lambda}\right) \\
\operatorname{var}\left(\hat{\gamma}_{\text {naive }}\right) & =O\left(\frac{1}{n h}+\frac{1}{n h_{z}}\right) .
\end{aligned}
$$

For the sharp design (9), the same results apply. The exact expressions for bias and variance are given in the appendix.

From this result it can be seen that the fastest rate of convergence possible for $\hat{\gamma}_{\text {naive }}$ by appropriate bandwidth choices is $n^{-\frac{1}{3}}$. (In the special case where the density is continuous, that is, $f^{-}\left(x \mid z_{0}\right)=f^{+}\left(x \mid z_{0}\right)$, the bias term with respect to the bandwidth $h$ is $O\left(h^{2}\right)$ such that a convergence rate of $n^{-\frac{2}{5}}$ is possible. In this article, we focus on the estimator proposed in the next section, though, because it can obtain $n^{-\frac{2}{5}}$ rate irrespective of whether the density is continuous or not.) It is straightforward to show asymptotic normality for this estimator, but the (first-order) approximation may not be very useful in practice as it would be dominated by the bias and variance terms $O(h)$ and $O\left(\frac{1}{n h}\right)$. The terms corresponding to the estimation error of $\hat{m}^{+}\left(x, z_{0}\right), \hat{m}^{-}\left(x, z_{0}\right), \hat{d}^{+}\left(x, z_{0}\right), \hat{d}^{-}\left(x, z_{0}\right)$ would be of lower order and thus ignored in the first-order approximation. The bias and variance approximation thus obtained would be the same as in a situation where $m^{+}\left(x, z_{0}\right), m^{-}\left(x, z_{0}\right), d^{+}\left(x, z_{0}\right), d^{-}\left(x, z_{0}\right)$ were known and not estimated. Hence, such an approximation might not be very accurate in small samples. A more useful approximation can be obtained by retaining also the lower order terms. However, it seems more promising to use $\hat{\gamma}_{\mathrm{RDD}}$ instead.

\subsection{Properties of $\hat{\gamma}_{\mathrm{RDD}}$}

The estimator $\hat{\gamma}_{\mathrm{RDD}}$ is based on (13), but uses the boundary kernel (15) in the second smoothing step, instead of (14). It thereby attains the convergence rate of a one-dimensional nonparametric regression estimator, irrespective of the dimension of $X$. It thus obtains the fastest convergence rate possible and is not affected by a curse of dimensionality. This is achieved by smoothing over all regressors $X$ and by an implicit boundary adaptation with respect to $Z$. (In addition, the bias and variance terms due to estimating $\mathrm{m}^{+}, \mathrm{m}^{-}, d^{+}, d^{-}$and due to estimating the density functions $\frac{f^{-}\left(x \mid z_{0}\right)+f^{+}\left(x \mid z_{0}\right)}{2}$ by the empirical distribution functions converge at the same rate.)

We derive the asymptotic distribution of this estimator and show that the asymptotic variance becomes smaller the more covariates $X$ are included. For the optimal convergence result further below, we need to be specific about the choice of the bandwidth values.

Assumption 3. The bandwidths satisfy the following conditions:

$$
\begin{aligned}
\lim _{n \rightarrow \infty} \sqrt{n h^{5}} & =r<\infty \\
\lim _{n \rightarrow \infty} \frac{h_{z}}{h} & =r_{z} \quad \text { with } 0<r_{z}<\infty \\
\lim _{n \rightarrow \infty} \frac{h_{x}^{\lambda / 2}}{h} & =r_{x}<\infty .
\end{aligned}
$$


This assumption ensures that the bias and standard deviation of the estimator converge at rate $n^{-\frac{2}{5}}$ to zero, that is, at the rate of a univariate nonparametric regression. Note that the last condition of Assumption 3 provides an upper bound on $h_{x}$, whereas Assumption (2iv) provides a lower bound on $h_{x}$. Suppose that $h_{x}$ depends on the sample size in the following way:

$$
h_{x} \propto n^{\zeta},
$$

then the bandwidth conditions of Assumptions 2 and 3 together require that

$$
-\frac{4}{5 L}<\zeta \leq-\frac{2}{5 \lambda}
$$

This implies that $h_{x}$ converges at a slower rate to zero than $h$ and $h_{z}$ when $L \geq 4$, that is, when $X$ contains four or more continuous regressors. Therefore, a necessary condition for Assumptions 2 and 3 to hold jointly is that $-\frac{4}{5 L}<-\frac{2}{5 \lambda}$ or equivalently $\lambda>\frac{L}{2}$. As further discussed below, this requires higher-order kernels if $X$ contains four or more continuous regressors, where as conventional kernels are sufficient otherwise. Assumption 3 is sufficient for the bias and variance to converge at the univariate nonparametric rate, which is summarized in the following theorem.

Theorem 2 (Asymptotic distribution of $\hat{\gamma}_{R D D}$ ).

(a) Under Assumptions 1 and 2, the bias and variance terms of $\hat{\gamma}_{\mathrm{RDD}}$, which is the estimator (13) with kernel function (15), are of order

$$
\begin{aligned}
\operatorname{Bias}\left(\hat{\gamma}_{\mathrm{RDD}}\right) & =O\left(h^{2}+h_{z}^{2}+h_{x}^{\lambda}\right) \\
\operatorname{var}\left(\hat{\gamma}_{\mathrm{RDD}}\right) & =O\left(\frac{1}{n h}+\frac{1}{n h_{z}}\right)
\end{aligned}
$$

(b) Under Assumptions 1, 2, and 3, the estimator is asymptotically normally distributed and converges at the univariate nonparametric rate

$$
\sqrt{n h}\left(\hat{\gamma}_{\mathrm{RDD}}-\gamma\right) \rightarrow N\left(\mathcal{B}_{\mathrm{RDD}}, \mathcal{V}_{\mathrm{RDD}}\right),
$$

where $\mathcal{B}_{\mathrm{RDD}}$

$$
\begin{aligned}
= & \frac{r}{\Gamma} \frac{\bar{\mu}_{2}^{2}-\bar{\mu}_{1} \bar{\mu}_{3}}{4 \tilde{\mu} f\left(z_{0}\right)} \int\left(m^{+}\left(x, z_{0}\right)-m^{-}\left(x, z_{0}\right)-\gamma\left(d^{+}\left(x, z_{0}\right)\right.\right. \\
& \left.\left.-d^{-}\left(x, z_{0}\right)\right)\right)\left(\frac{\partial^{2} f^{+}}{\partial z^{2}}\left(x, z_{0}\right)+\frac{\partial^{2} f^{-}}{\partial z^{2}}\left(x, z_{0}\right)\right) d x \\
& +\frac{r r_{z}^{2}}{\Gamma} \frac{\bar{\mu}_{2}^{2}-\bar{\mu}_{1} \bar{\mu}_{3}}{2 \tilde{\mu}} \int\left(\frac{\partial^{2} m^{+}\left(x, z_{0}\right)}{\partial z^{2}}-\frac{\partial^{2} m^{-}\left(x, z_{0}\right)}{\partial z^{2}}\right. \\
& \left.-\gamma \frac{\partial^{2} d^{+}\left(x, z_{0}\right)}{\partial z^{2}}+\gamma \frac{\partial^{2} d^{-}\left(x, z_{0}\right)}{\partial z^{2}}\right) \frac{f^{-}\left(x, z_{0}\right)+f^{+}\left(x, z_{0}\right)}{2 f\left(z_{0}\right)} d x \\
& +\frac{r r_{x}^{2} \eta_{\lambda}}{\Gamma} \int \sum_{l=1}^{L}\left\{\frac{\partial^{\lambda} m^{+}\left(x, z_{0}\right)}{\lambda ! \cdot \partial x_{l}^{\lambda}}+\sum_{s=1}^{\lambda-1} \frac{\partial^{s} m^{+}\left(x, z_{0}\right)}{\partial x_{l}^{s}} \omega_{s}^{+}\right. \\
& \left.-\frac{\partial^{\lambda} m^{-}\left(x, z_{0}\right)}{\lambda ! \cdot \partial x_{l}^{\lambda}}-\sum_{s=1}^{\lambda-1} \frac{\partial^{s} m^{-}\left(x, z_{0}\right)}{\partial x_{l}^{s}} \omega_{s}^{-}\right\} \frac{f^{-}\left(x, z_{0}\right)+f^{+}\left(x, z_{0}\right)}{2 f\left(z_{0}\right)} d x \\
& -\frac{\gamma r r_{x}^{2} \eta_{\lambda}}{\Gamma} \int \sum_{l=1}^{L}\left\{\frac{\partial^{\lambda} d^{+}\left(x, z_{0}\right)}{\lambda ! \cdot \partial x_{l}^{\lambda}}+\sum_{s=1}^{\lambda-1} \frac{\partial^{s} d^{+}\left(x, z_{0}\right)}{\partial x_{l}^{s}} \omega_{s}^{+}\right. \\
& \left.-\frac{\partial^{\lambda} d^{-}\left(x, z_{0}\right)}{\lambda ! \cdot \partial x_{l}^{\lambda}}-\sum_{s=1}^{\lambda-1} \frac{\partial^{s} d^{-}\left(x, z_{0}\right)}{\partial x_{l}^{s}} \omega_{s}^{-}\right\} \frac{f^{-}\left(x, z_{0}\right)+f^{+}\left(x, z_{0}\right)}{2 f\left(z_{0}\right)} d x,
\end{aligned}
$$

where $\quad \Gamma=\int\left(d^{+}\left(x, z_{0}\right)-d^{-}\left(x, z_{0}\right)\right) \cdot \frac{f^{-}\left(x \mid z_{0}\right)+f^{+}\left(x \mid z_{0}\right)}{2} d x$ and $\quad \omega_{s}^{+}=\left\{\frac{\partial^{\lambda-s} f^{+}\left(x_{i}, z_{0}\right)}{s !(\lambda-s) ! \cdot \cdot x_{l}^{\lambda-s}}-\frac{\partial^{\lambda-1} f^{+}\left(x_{0}, z_{0}\right)}{\partial x_{1}^{\lambda-1}} \cdot\left(\frac{\partial^{\lambda-2} f^{+}\left(x_{0}, z_{0}\right)}{\partial x_{l}^{\lambda-2}}\right)^{-1}\right.$ $\left.\frac{(\lambda-2) !}{(\lambda-1) ! s !(\lambda-1-s) !} \frac{\partial^{\lambda-1-s} f^{+}\left(x_{i}, z_{0}\right)}{\partial x_{l}^{\lambda-1-s}}\right\} / f^{+}\left(x_{i}, z_{0}\right)$ and $\omega_{s}^{-}$defined analogously

and $\mathcal{V}_{\mathrm{RDD}}$

$$
\begin{aligned}
= & \frac{\bar{\mu}_{2}^{2} \ddot{\mu}_{0}-2 \bar{\mu}_{2} \bar{\mu}_{1} \ddot{\mu}_{1}+\bar{\mu}_{1}^{2} \ddot{\mu}_{2}}{\Gamma^{2} 4 \tilde{\mu}^{2} f^{2}\left(z_{0}\right)} \times\left(\frac{1}{r_{z}} \int\left(f^{+}\left(x, z_{0}\right)+f^{-}\left(x, z_{0}\right)\right)^{2}\right. \\
& \times\left(\frac{\sigma_{Y}^{2+}\left(x, z_{0}\right)-2 \gamma \sigma_{Y D}^{2+}\left(x, z_{0}\right)+\gamma^{2} \sigma_{D}^{2+}\left(x, z_{0}\right)}{f^{+}\left(x, z_{0}\right)}\right. \\
& \left.+\frac{\sigma_{Y}^{2-}\left(x, z_{0}\right)-2 \gamma \sigma_{Y D}^{2-}\left(x, z_{0}\right)+\gamma^{2} \sigma_{D}^{2-}\left(x, z_{0}\right)}{f^{-}\left(x, z_{0}\right)}\right) d x \\
& +\int\left\{m^{+}\left(x, z_{0}\right)-\gamma d^{+}\left(x, z_{0}\right)-m^{-}\left(x, z_{0}\right)+\gamma d^{-}\left(x, z_{0}\right)\right\}^{2} \\
& \left.\cdot\left(f^{+}\left(x, z_{0}\right)+f^{-}\left(x, z_{0}\right)\right) d x\right)
\end{aligned}
$$

where $\quad \sigma_{Y}^{2+}(X, z)=\lim _{\varepsilon \rightarrow 0} E\left[\left(Y-m^{+}(X, Z)\right)^{2} \mid X, Z=\right.$ $z+\varepsilon]$ and $\sigma_{Y D}^{2+}(X, z)=\lim _{\varepsilon \rightarrow 0} E\left[\left(Y-m^{+}(X, Z)\right)(D-\right.$ $\left.\left.d^{+}(X, Z)\right) \mid X, Z=z+\varepsilon\right]$ and $\sigma_{D}^{2+}(X, z)=\lim _{\varepsilon \rightarrow 0} E[(D-$ $\left.\left.d^{+}(X, Z)\right)^{2} \mid X, Z=z+\varepsilon\right]$ and analogously for $\sigma_{Y}^{2+}$ $(X, z), \sigma_{Y D}^{2+}(X, z)$, and $\sigma_{D}^{2+}(X, z)$.

For the sharp design (9), the same results are obtained but the formulas are simpler. $d^{+}$and $d^{-}$are not estimated but set to 1 and 0 , respectively. This implies that $\Gamma=1$ and the terms $\sigma_{D}^{2+}$, $\sigma_{D}^{2-}, \sigma_{Y D}^{2+}, \sigma_{Y D}^{2-}$ and all derivatives of $d^{+}\left(x, z_{0}\right)$ and $d^{-}\left(x, z_{0}\right)$ are zero.

Note that Assumption 3 is stronger than needed for the results of Theorem 3. For obtaining $n^{-\frac{2}{5}}$ convergence weaker rate conditions would suffice. In other words, it would not be needed that the ratios of the bandwidths converge to a welldefined limit point. Assumption 3 permits obtaining concise and explicit expressions for bias and variance, though. We also see that undersmoothing is permitted: For a choice of $r=0$ in Assumption 3, the limit bias term is zero, that is, $\mathcal{B}_{\mathrm{RDD}}=0$. Such undersmoothing is convenient, for example, for developing test statistics. (We thank a referee for pointing this out.)

Part (18) of Assumption 3 requires that $\lambda>\frac{L}{2}$ to control the bias due to smoothing in the $X$ dimension. If $X$ contains at most three continuous regressors, a second order kernel $\lambda=2$ can be used. Otherwise, higher order kernels are required to achieve an $n^{-\frac{2}{5}}$ convergence rate. Instead of using higher order kernels, one could alternatively use local higher order polynomial regression instead of local linear regression (16). However, when the number of regressors in $X$ is large, this could be inconvenient to implement in practice since a large number of interaction and higher order terms would be required, which could give rise to problems of local multicollinearity in small samples and/or for small bandwidth values. On the other hand, higher order kernels are very convenient to implement when a product kernel (17) is used. Higher order kernels are only necessary for smoothing in the $X$ dimension but not for smoothing along $Z$. 
When a second-order kernel is used and $X$ contains at most three continuous regressors, the bias term $\mathcal{B}_{\mathrm{RDD}}$ simplifies to

$$
\begin{aligned}
& \frac{r}{\Gamma} \frac{\bar{\mu}_{2}^{2}-\bar{\mu}_{1} \bar{\mu}_{3}}{4 \tilde{\mu} f\left(z_{0}\right)} \int\left(m^{+}\left(x, z_{0}\right)-m^{-}\left(x, z_{0}\right)-\gamma\left(d^{+}\left(x, z_{0}\right)\right.\right. \\
& \left.\left.\quad-d^{-}\left(x, z_{0}\right)\right)\right)\left(\frac{\partial^{2} f^{+}}{\partial z^{2}}\left(x, z_{0}\right)+\frac{\partial^{2} f^{-}}{\partial z^{2}}\left(x, z_{0}\right)\right) d x \\
& \quad+\frac{r r_{z}^{2}}{\Gamma} \frac{\bar{\mu}_{2}^{2}-\bar{\mu}_{1} \bar{\mu}_{3}}{2 \tilde{\mu}} \int\left(\frac{\partial^{2} m^{+}\left(x, z_{0}\right)}{\partial z^{2}}-\frac{\partial^{2} m^{-}\left(x, z_{0}\right)}{\partial z^{2}}\right. \\
& \left.\quad-\gamma \frac{\partial^{2} d^{+}\left(x, z_{0}\right)}{\partial z^{2}}+\gamma \frac{\partial^{2} d^{-}\left(x, z_{0}\right)}{\partial z^{2}}\right) \cdot \frac{f^{-}\left(x, z_{0}\right)+f^{+}\left(x, z_{0}\right)}{2 f\left(z_{0}\right)} d x \\
& \quad+\frac{r r_{x}^{2} \mu_{2}}{2 \Gamma} \int \sum_{l=1}^{L}\left\{\frac{\partial^{2} m^{+}\left(x, z_{0}\right)}{\partial x_{l}^{2}}-\frac{\partial^{2} m^{-}\left(x, z_{0}\right)}{\partial x_{l}^{2}}\right. \\
& \left.\quad-\gamma \frac{\partial^{2} d^{+}\left(x, z_{0}\right)}{2 \cdot \partial x_{l}^{2}}+\gamma \frac{\partial^{2} d^{-}\left(x, z_{0}\right)}{2 \cdot \partial x_{l}^{2}}\right\} \cdot \frac{f^{-}\left(x, z_{0}\right)+f^{+}\left(x, z_{0}\right)}{2 f\left(z_{0}\right)} d x .
\end{aligned}
$$

It remains to be discussed how the bandwidth values $h, h_{z}$, and $h_{x}$ should be chosen in practice. It is beyond the scope of this article to develop a data-driven bandwidth selector, and we therefore limit ourselves to a procedure that is rate optimal, that is, satisfies Assumptions 2 and 3 as $n$ increases to infinity. The first part of Assumption 3 suggests to choose $h$ proportional to $n^{-\frac{1}{5}}$, which corresponds to the rate for univariate nonparametric regression. A simple procedure is to choose $h$ via (least-square) cross-validation with respect to a nonparametric regression of $Y$ on $Z$ (outside of a neighborhood around $z_{0}$ ), which is known to provide a bandwidth that converges at the desired rate. (At the same time it is known that the bandwidth obtained by crossvalidation converges only very slowly to the true optimal bandwidth. Nevertheless, many applied researchers proceed by using the bandwidth obtained from cross-validation and then examine the sensitivity of the final estimation results to changes in the bandwidth values by reestimating with various multiples and/or fractions of the original bandwidth values.)

With an estimate for $h$, we can choose $h_{z}=h$ which is permitted by Assumptions 2 and 3. If $X$ contains at most three continuous regressors, we can also choose $h_{x}=h$. On the other hand, if $L \geq 4$, then $h_{x}$ should converge at a slower rate than $h$ and $h_{z}$. Assumptions 2 and 3 give us some leeway in the exact choice of $h_{x}$. If we would like to make the bias small (for reasons discussed in the next section), we would choose the lower bound of (18) to set $h_{x}=c_{1} \cdot n^{-\frac{4}{5 L}+\delta}$ for a small positive $\delta$ and some positive constant $c_{1}$. This contrasts with the choice for $h$ which is given as $h=c_{2} \cdot n^{-\frac{1}{5}}$. We do not know the optimal $c_{1}$ and $c_{2}$, but since we only aim for a rate optimal choice, we can set $c_{1}=c_{2}$ to obtain $h_{x}=c_{1} \cdot n^{-\frac{4}{5 L}+\delta}=c_{1} n^{-\frac{4}{5 L}+\delta} \cdot n^{\frac{1}{5}} n^{-\frac{1}{5}}$ such that

$$
h_{x}=n^{\frac{1-4 / L+5 \delta}{5}} \cdot h .
$$

We can thus use the bandwidth $h$ obtained via cross-validation and multiply it with $n^{\frac{1-4 / L+5 \delta}{5}}$ for some small $\delta$ to obtain the (larger) bandwidth value for $h_{x}$. Having estimated $\hat{\gamma}_{\mathrm{RDD}}$ with these bandwidths, one would usually examine the robustness of the results to the bandwidths values.

\subsection{Variance Reduction Through the Use of Control Variables}

In most of the discussion so far, it was permitted that $f(x \mid z)$ is discontinuous at $z_{0}$ such that controlling for $X$ allows reducing bias. In the case where $f(x \mid z)$ is continuous, controlling for $X$ is still helpful: It can reduce the variance of the estimator, which is shown in the following theorem. Suppose that the covariates are identically distributed on both sides of the threshold (i.e., $f(x \mid z)$ is continuous) such that $\gamma$ is identified with and without controlling for any $X$. In this case, one could use $\hat{\gamma}_{\text {RDD }}$ with $X$ being the empty set. This estimator is henceforth denoted as $\hat{\gamma}_{n o X}$. Alternatively, one could use a set of control variables $X$ in the estimator, which we denote as $\hat{\gamma}_{\text {RDD }}$ as before. Suppose that both estimators are consistent for $\gamma$. As shown below, $\hat{\gamma}_{\text {noX }}$ generally has a larger asymptotic variance than $\hat{\gamma}_{\text {RDD }}$. (We would like to point out that the result in Theorem 4 only refers to the variance. While we find that covariates reduce variance, we do not have a corresponding result for the bias. Hence, in certain situations, asymptotic bias could possibly increase and we, therefore, cannot rule out that the inclusion of covariates $X$ in certain cases could even increase MSE if in such situations an increase in squared bias is larger than the decrease of variance due to the inclusion of $X$.) On the other hand, an ordering of squared biases seems impossible under general conditions. However, by Assumption 3 we can set $r=0$, that is, choose a bandwidth sequence such that the ratio of the squared bias to variance converges to zero. Such undersmoothing implies that the asymptotic bias $\mathcal{B}_{\mathrm{RDD}}$ is zero and the mean squared error is thus identical to $\mathcal{V}_{\mathrm{RDD}}$. With such undersmoothing, we only need to analyze the asymptotic variance. As outlined below, there are precision gains by controlling for $X$ even if the RDD estimator would be consistent without covariates.

For stating Theorem 3 in a concise way, some further notation is required. Let $w^{+}(X, z)=\lim _{\varepsilon \rightarrow 0} E[Y-\gamma D \mid X, Z=z+\varepsilon]$ be the right limit of the difference between $Y$ and $\gamma D$, and $w^{+}(z)=\lim _{\varepsilon \rightarrow 0} E[Y-\gamma D \mid Z=z+\varepsilon]$ be the corresponding expression without conditioning on $X$. (This also contains the sharp design (9) as a special case, where $\quad w^{+}(X, z)=\lim _{\varepsilon \rightarrow 0} E[Y-\gamma \mid X, Z=z+\varepsilon] \quad$ and $w^{-}(X, z)=\lim _{\varepsilon \rightarrow 0} E[Y \mid X, Z=z-\varepsilon]$.) Define the variance of $w^{+}\left(X, z_{0}\right)$ as $V^{+}=\int\left\{w^{+}\left(x, z_{0}\right)-w^{+}\left(z_{0}\right)\right\}^{2} f\left(x \mid z_{0}\right) d x$. Define $w^{-}(X, z), w^{-}(z)$ and $V^{-}$analogously as the left limits. Theorem 3 shows that there is a reduction in variance if $V^{+} \neq 0$ and/or $V^{-} \neq 0$.

To gain some intuition, note that $V^{+}$is the variance of the conditional expectation of $Y$ given $X$ plus the variance of the conditional expectation of $\gamma D$ given $X$ minus the covariance between these two terms. Hence, $V^{+}$is usually nonzero if $X$ is a predictor of $Y$ and/or of $D$. On the other hand, $V^{+}$and $V^{-}$are zero only if $X$ neither predicts $Y$ nor $D$. (This discussion excludes the unreasonable case where it predicts both but not $Y-\gamma D$.) Define further the covariance $C$ as $\int\left(w^{+}\left(x, z_{0}\right)-w^{+}\left(z_{0}\right)\right)\left(w^{-}\left(x, z_{0}\right)-\right.$ $\left.w^{-}\left(z_{0}\right)\right) f\left(x \mid z_{0}\right) d x$. For the case where $V^{+}$and $V^{-}$are both nonzero, we define the correlation coefficient $R=\frac{C}{\sqrt{V^{+} V^{-}}}$. Now, we can state the result in terms of the variances and the correlation coefficient, which also depends on the bandwidth sequences. The variance of $\hat{\gamma}_{\text {RDD }}$ is a function of smoothing in the $Z$ dimension via $h$ and $h_{z}$. The $\hat{\gamma}_{n o X}$ estimator only depends 
on $h_{z}$ since there is no smoothing in the second step. A natural choice would thus be $h=h_{z}$. (The variance of $\hat{\gamma}_{\mathrm{RDD}}$ can be reduced even further relative to $\hat{\gamma}_{\text {noX }}$ by choosing $h_{z}<h$, but this would be more of a technical trick than a substantive result.) This implies $r_{z}=1$ in Assumption 3. Using this notation, the difference in the asymptotic variances can be written as

$$
\begin{aligned}
\mathcal{V}_{\mathrm{RDD}}-\mathcal{V}_{\text {noX }}= & \left\{\frac{r_{z}-2}{2} V^{+}+\frac{r_{z}-2}{2} V^{-}-r_{z} C\right\} \\
& \times\left(\frac{\bar{\mu}_{2}^{2} \ddot{\mu}_{0}-2 \bar{\mu}_{2} \bar{\mu}_{1} \ddot{\mu}_{1}+\bar{\mu}_{1}^{2} \ddot{\mu}_{2}}{\Gamma^{2} \tilde{\mu}^{2} f\left(z_{0}\right) r_{z}}\right)
\end{aligned}
$$

or, if $V^{+}$and $V^{-}$are both nonzero, as $=\left\{\frac{r_{z}-2}{2} V^{+}+\frac{r_{z}-2}{2} V^{-}-\right.$ $\left.r_{z} R \sqrt{V^{+} V^{-}}\right\}\left(\frac{\bar{\mu}_{2}^{2} \ddot{\mu}_{0}-2 \bar{\mu}_{2} \bar{\mu}_{1} \ddot{\mu}_{1}+\bar{\mu}_{1}^{2} \ddot{\mu}_{2}}{\Gamma^{2} \tilde{\mu}^{2} f\left(z_{0}\right) r_{z}}\right)$, as derived in the appendix. This implies the following.

Theorem 3. Let $\hat{\gamma}_{\mathrm{RDD}}$ be the estimator (13) with kernel function (15) using the set of regressors $X$, and let $\hat{\gamma}_{n o X}$ be the estimator with $X$ being the empty set. Denote the asymptotic variance of $\hat{\gamma}_{n o X}$ by $\mathcal{V}_{\text {noX }}$ and assume that both estimators consistently estimate $\gamma$ and satisfy Assumptions 2 and 3. Assume further that the distribution of $X$ is continuous at $z_{0}$, that is, $f^{+}\left(X, z_{0}\right)=f^{-}\left(X, z_{0}\right)$ a.s.

(a) If $V^{+}=V^{-}=0$ then

$$
\mathcal{V}_{\mathrm{RDD}}=\mathcal{V}_{\text {noX }}
$$

(b) Under any of the following conditions

$$
\mathcal{V}_{\mathrm{RDD}}<\mathcal{V}_{\text {noX }}
$$

- if $V^{+}=0$ and $V^{-} \neq 0$ or vice versa and $r_{z}<2$

- or if $V^{+} \neq 0$ and $V^{-} \neq 0$ and $R \geq 0$ and $r_{z}<2$

- or if $V^{+} \neq 0$ and $V^{-} \neq 0$ and $-1<R<0$ and $r_{z}<2 \frac{1+R}{1-R^{2}}$.

- or if $V^{+} \neq 0$ and $V^{-} \neq 0$ and $R=-1$ and $r_{z}<1$.

Hence, if, in case (a) of Theorem 3, where $X$ has no predictive power neither for $Y$ nor for $D$, the asymptotic variances are the same. On the other hand, if $X$ has predictive power either for $Y$ or for $D$ and one uses the same bandwidths for both estimators $\left(h_{z}=h\right)$, the RDD estimator with covariates has a strictly smaller variance. (In the sharp design (9), $X$ cannot have predictive power for $D$ (conditional on $Z$ ), hence predictive power for $Y$ is needed.) This holds in all cases except for the very implausible scenario where $w^{+}\left(X, z_{0}\right)$ and $w^{-}\left(X, z_{0}\right)$ are negatively correlated with a correlation coefficient of -1 . In most economic applications, however, one would rather expect a positive correlation.

( $\hat{\gamma}_{\text {RDD }}$ has a smaller variance than $\hat{\gamma}_{n o X}$ as it exploits the available information more effectively. Consider, for simplicity, the sharp design. $\hat{\gamma}_{n o X}$ estimates the conditional mean of $Y$ left and right of the threshold. In terms of iterated expectations, the left limit of the mean of $Y$ at the threshold could be estimated as the left limit of the mean of $Y$ conditional on $X$ averaged out with respect to the distribution of $X$, using only data points to the left of the threshold. In contrast, $\hat{\gamma}_{\text {RDD }}$ estimates the left limit of the mean of $Y$ conditional on $X$, but then takes averages with respect to the distribution of $X$ in the neighborhood of $z_{0}$. In the case where the distribution of $X$ is continuous at $z_{0}$, that is,
$f^{+}\left(X, z_{0}\right)=f^{-}\left(X, z_{0}\right)$, the estimator $\hat{\gamma}_{\text {RDD }}$ uses the data points $X_{i}$ in the left and in the right neighborhood of $z_{0}$ to estimate $f\left(X, z_{0}\right)$, whereas $\hat{\gamma}_{\text {noX }}$ uses only the data on one side of the threshold. This implies that $\hat{\gamma}_{\text {RDD }}$ uses more information in the estimation of the empirical distribution function $F\left(X, z_{0}\right)$, which leads to the variance reductions in Theorem 3.

Theorem 3 can easily be extended to show that the RDD estimator with a larger regressor set $\mathbf{X}$, that is, where $X \subset \mathbf{X}$, has smaller asymptotic variance than the RDD estimator with $X$. (The proof is analogous and is omitted.) Hence, one can combine specific covariates for eliminating bias with adding further covariates to reduce variance. The more variables are included in $\mathbf{X}$ the smaller the variance will be.)

\section{SIMULATIONS}

This section presents a simulation study to investigate the finite sample performance of the suggested method in the context of the sharp and fuzzy RDD. Starting with the former, we consider the following data-generating process (DGP):

$$
\begin{aligned}
& Z, U, V, W \sim \mathcal{N}(0,1) \text { independently of each other, } \\
& D=I\{Z>0\}, \quad X_{1}=\alpha D+0.5 U, \quad X_{2}=\alpha D+0.5 \mathrm{~V}, \\
& Y=D+0.5 Z-0.25 D Z+0.25 Z^{2}+\beta\left(X_{1}+X_{2}\right) \\
& +\frac{\beta}{2}\left(X_{1}^{2}+X_{2}^{2}\right)+W \text {. }
\end{aligned}
$$

Both the running variable $Z$ and the unobservables $U, V, W$, which affect the covariates $X_{1}, X_{2}$ and the outcome $Y$, respectively, are standard normally distributed. The parameter $\alpha$ reflects the strength of the association between the distributions of $X_{1}, X_{2}$ and the treatment state $D . \beta$ determines the impact of $X_{1}, X_{2}$ and their higher order terms on $Y$. In the simulations, we consider various combinations of $\alpha$ and $\beta$. First, we set $\alpha=0$ and $\beta=0.4$ such that the covariates affect the outcome, but are balanced around the threshold. In this case, controlling for $X=\left(X_{1}, X_{2}\right)$ is not necessary for the consistency of RDD, but might reduce the variance. Second, we set $\alpha=0.2$ and $\beta=0.4$, implying that the distribution of $X$ differs across treatment states at the threshold and that $X$ affects $Y$.

We run 1000 simulations and consider sample sizes of $n=1000$ and 4000 to analyze RDD estimation based on the boundary kernel $\hat{\gamma}_{\mathrm{RDD}}$, see (15). Least-square cross-validation $(\mathrm{CV})$ is used to select the bandwidths for the estimation of $m^{+}(x, z)$ and $m^{-}(x, z)$ (using local linear regression) as well as $K_{h}(u)$ required in $(13)$, (For $m^{+}(X, Z)$ and $m^{-}(X, Z)$, the bandwidth selector $\mathrm{CV}$ only uses treated and nontreated observations, respectively.) based on the np package for the statistical software R by Hayfield and Racine (2008). In addition, we also make use of undersmoothing and oversmoothing by taking half or twice the $\mathrm{CV}$ bandwidth, respectively $(\mathrm{CV} / 2,2 \mathrm{CV})$.

We compare our method to conventional RDD estimation without covariates as implemented in the rdd package for $\mathrm{R}$ by Dimmery (2016), which is based on a local linear regression of $Y$ on $Z$. We consider several bandwidths choices, namely, the values picked by the $\mathrm{CV}$ procedure for $\hat{\gamma}_{\mathrm{RDD}}$; the method of Imbens and Kalyanaraman (2012) (IK) for optimal bandwidth selection in RDD; the robust inference approach of Calonico, Cattaneo, 
Table 1. Simulations—-sharp RDD

\begin{tabular}{|c|c|c|c|c|c|c|c|c|c|c|c|c|c|c|}
\hline Bandwidth & $\mathrm{CV}$ & $\mathrm{CV} / 2$ & $2 \mathrm{CV}$ & $\mathrm{CV}$ & IK & CCT & LM & $\mathrm{CV}$ & $\mathrm{CV} / 2$ & $2 \mathrm{CV}$ & $\mathrm{CV}$ & IK & CCT & LM \\
\hline bias & 0.00 & -0.00 & 0.00 & -0.00 & 0.01 & 0.00 & -0.00 & -0.00 & -0.00 & -0.00 & -0.01 & -0.00 & -0.01 & -0.00 \\
\hline sdev & 0.15 & 0.27 & 0.13 & 0.43 & 0.20 & 0.22 & 0.15 & 0.09 & 0.12 & 0.09 & 0.27 & 0.10 & 0.10 & 0.08 \\
\hline$\alpha=0.2, \beta=0.4$ & \multicolumn{7}{|c|}{$n=1000$} & \multicolumn{7}{|c|}{$n=4000$} \\
\hline bias & -0.00 & -0.00 & -0.01 & 0.18 & 0.18 & 0.18 & 0.17 & -0.00 & -0.00 & -0.01 & 0.16 & 0.17 & 0.17 & 0.17 \\
\hline sdev & 0.17 & 0.27 & 0.14 & 0.45 & 0.20 & 0.22 & 0.15 & 0.09 & 0.13 & 0.09 & 0.30 & 0.10 & 0.10 & 0.08 \\
\hline rmse & 0.17 & 0.27 & 0.14 & 0.48 & 0.27 & 0.28 & 0.23 & 0.09 & 0.13 & 0.09 & 0.34 & 0.20 & 0.20 & 0.19 \\
\hline
\end{tabular}

NOTE: "CV," "CV/2," "2CV" stands for bandwidth selection based on least-square cross-validation, as well as twice and half that value. "IK" is the optimal Imbens-Kalyanaraman (2012) bandwidth. "CCT" is the robust inference approach of Calonico, Cattaneo, and Titiunik (2014) (CCT). "LM" is the local cross-validation approach of Ludwig and Miller (2007) based on the median values of the running variable above and below the threshold. "bias," "sdev," and "rmse" report the bias, standard deviation, and root mean squared error of the respective method.

and Titiunik (2014) (CCT) as implemented as default option in the rdrobust package for $\mathrm{R}$ by Calonico, Cattaneo, and Titiunik (2015); and the local cross-validation approach of Ludwig and Miller (2007) (LM) based on the median values of the running variable above and below the threshold. In all estimations, the Epanechnikov kernel is used.

Table 1 reports the bias, standard deviation, and root mean squared error (RMSE) of the estimators for various choices of $\alpha, \beta$ in the sharp RDD. When setting $\alpha=0, \beta=0.4$, all procedures are unbiased as expected. Under either sample size, $\hat{\gamma}_{\mathrm{RDD}}$ outperforms RDD without $X$ in terms of precision when using the same CV bandwidth for both estimators. Furthermore, $\hat{\gamma}_{\mathrm{RDD}}$ with $\mathrm{CV}$ is in most cases also more precise than RDD without $X$ based on the IK, CCT, and LM bandwidths. (Under $n=1000, \alpha=0, \beta=0.4$, the means (standard deviations) of the $\mathrm{CV}$, IK, CCT, and LM bandwidths for $Z$ are 0.16 (0.06), 0.84 (0.29), 0.66 (0.11), 1.58 (0.51), respectively. The means and standard deviations are very similar under $n=$ $1000, \alpha=0.2, \beta=0.4$.) As expected, a smaller bandwidth $(\mathrm{CV} / 2)$ increases the standard deviation of $\hat{\gamma}_{\mathrm{RDD}}$, while a larger bandwidth (2CV) slightly decreases it. For $n=4000$, however, the differences in precision are quite moderate for various bandwidth choices.

When setting $\alpha=0.2$ and $\beta=0.4$, the biases of $\hat{\gamma}_{\mathrm{RDD}}$ are again close to zero, while this is no longer the case for RDD without $X$. For $n=1000, \hat{\gamma}_{\mathrm{RDD}}$ with $\mathrm{CV}$ and $2 \mathrm{CV}$ dominates any RDD without $X$ in terms of bias, standard deviation, and root mean squared error (RMSE), while $\hat{\gamma}_{\mathrm{RDD}}$ with $\mathrm{CV} / 2$ is less precise. Under $n=4000$, all three versions of $\hat{\gamma}_{\mathrm{RDD}}$ have a considerably smaller RMSE than any RDD without $X$.

Second, we consider the case of a fuzzy RDD. We modify the DGP by replacing $D=I\{Z>0\}$ in (19) with $D=I\{-1+$ $2 I\{Z>0\}+0.5 U+Q>0\}$, with $Q \sim \mathcal{N}(0,1)$ independently of any other variable. $D$ is now endogenous even at the threshold due to $U$ entering both the treatment and outcome equation. The bandwidths used for the estimation of $d^{+}(x, z)$ and $d^{-}(x, z)$ required for the fuzzy RDD method are selected in an analogous way as for $m^{+}(x, z)$ and $m^{-}(x, z)$. We also consider fuzzy RDD estimation without covariates based on Dimmery (2016) with CV, IK, CCT, and LM bandwidth choices, respectively. (Under

Table 2. Simulations-fuzzy RDD

\begin{tabular}{|c|c|c|c|c|c|c|c|c|c|c|c|c|c|c|}
\hline Bandwidth & $\mathrm{CV}$ & $\mathrm{CV} / 2$ & $2 \mathrm{CV}$ & $\mathrm{CV}$ & IK & $\mathrm{CCT}$ & $\mathrm{LM}$ & $\mathrm{CV}$ & $\mathrm{CV} / 2$ & $2 \mathrm{CV}$ & $\mathrm{CV}$ & IK & $\mathrm{CCT}$ & LM \\
\hline bias & -0.01 & 0.00 & -0.02 & -0.05 & -0.02 & -0.01 & -0.01 & 0.01 & -0.00 & 0.01 & -0.01 & 0.00 & -0.01 & -0.01 \\
\hline sdev & 0.27 & 0.42 & 0.22 & 0.76 & 0.34 & 0.34 & 0.24 & 0.16 & 0.18 & 0.14 & 0.34 & 0.16 & 0.16 & 0.12 \\
\hline bias & -0.01 & -0.00 & -0.03 & 0.25 & 0.27 & 0.27 & 0.27 & 0.01 & 0.01 & 0.00 & 0.25 & 0.28 & 0.27 & 0.27 \\
\hline sdev & 0.28 & 0.52 & 0.23 & 0.67 & 0.33 & 0.34 & 0.23 & 0.15 & 0.20 & 0.15 & 0.39 & 0.16 & 0.16 & 0.12 \\
\hline rmse & 0.28 & 0.52 & 0.23 & 0.72 & 0.43 & 0.43 & 0.36 & 0.15 & 0.20 & 0.15 & 0.46 & 0.32 & 0.31 & 0.30 \\
\hline
\end{tabular}

NOTES: "CV," "CV/2," "2CV" stands for bandwidth selection based on least-square cross-validation, as well as twice and half that value. "IK" is the optimal Imbens-Kalyanaraman (2012) bandwidth. "CCT" is the robust inference approach of Calonico, Cattaneo, and Titiunik (2014) (CCT). "LM" is the local cross-validation approach of Ludwig and Miller (2007) based on the median values of the running variable above and below the threshold. "bias," "sdev," and "rmse" report the bias, standard deviation, and root mean squared error of the respective method. 
$n=1000, \alpha=0, \beta=0.4$, the means (standard deviations) of the CV, IK, CCT, and LM bandwidths for $Z$ are $0.23(0.07)$, 0.84 (0.29), $0.66(0.11), 1.73(0.59)$, respectively. The means and standard deviations are very similar under $n=1000, \alpha=$ $0.2, \beta=0.4$.) The results are reported in Table 2 and show a qualitatively similar pattern as for the sharp RDD. However, standard errors are generally larger as estimation is based on the compliers only, which by the definition of the DGP make up for about $65 \%$ of the population.

\section{APPLICATION}

As an empirical illustration of our method, we use data from Lalive (2008), who studies a labor market program introduced in June 1988 that extended the maximum duration of unemployment benefits from 30 to 209 weeks for job seekers aged 50 or older in certain regions of Austria under particular conditions. This suggests the use of a sharp RDD for assessing the program's effect on labor market outcomes such as unemployment duration. The treatment is defined based on the age threshold of 50. As acknowledged by Lalive (2008), however, a concern is that employees and companies could manipulate age at entry into unemployment, for example, by postponing a layoff in a way that the age requirement is just satisfied. This is a common concern in many applications. If such manipulations are selective with respect to employee characteristics that also affect labor market outcomes, conventional RDD without covariates fails to identify the effect of the program due to confounding related to an imbalance of the characteristics around the threshold. In contrast, our method remains consistent if all labor market relevant characteristics are plausibly observed in the data. As a word of caution, however, we would like to point out that this cannot be taken for granted in our application. For instance, unobserved individual characteristics like motivation, (dis-)utility from work, and self-confidence might predict both manipulation and labor market success. To consistently estimate the program effect by our method, it is required that these factors do not entail confounding conditional on the socioeconomic and employment-related characteristics available in the data (see the discussion below).

Our analysis makes use of the Austrian social security database, which includes information on job seekers (age, employment, unemployment, and earnings history) and the employers (region and industry), and the Austrian unemployment register, which contains information on the place of residence and socio-economic characteristics. The universe of inflows into unemployment between 1986 and 1995 is covered, and the inflow sample can be followed up until the end of 1998. We refer to Lalive (2008) for a description of sample adjustments made to the dataset. Specifically, we consider the female subsample in the age bracket 46 to 53 years living in a region where the program had been introduced, consisting of 5659 observations. The outcome variable $Y$ is unemployment duration, measured as weeks registered at the unemployment office. The running variable $Z$ is distance to the age threshold of 50 , measured in months divided by 12 . Table 3 reports sample means and balancing tests at the threshold for potentially labor market relevant characteristics, which serve as $X$. The tests are based on running RDD estimations with the elements in $X$ as outcome variables using the 'rdd' package, which performs local linear regression around the threshold. Estimates, standard errors, and $p$-values are reported for the IK bandwidth and half of it. Indeed, several covariates are imbalanced around the

Table 3. Covariate sample means and balance tests at the threshold

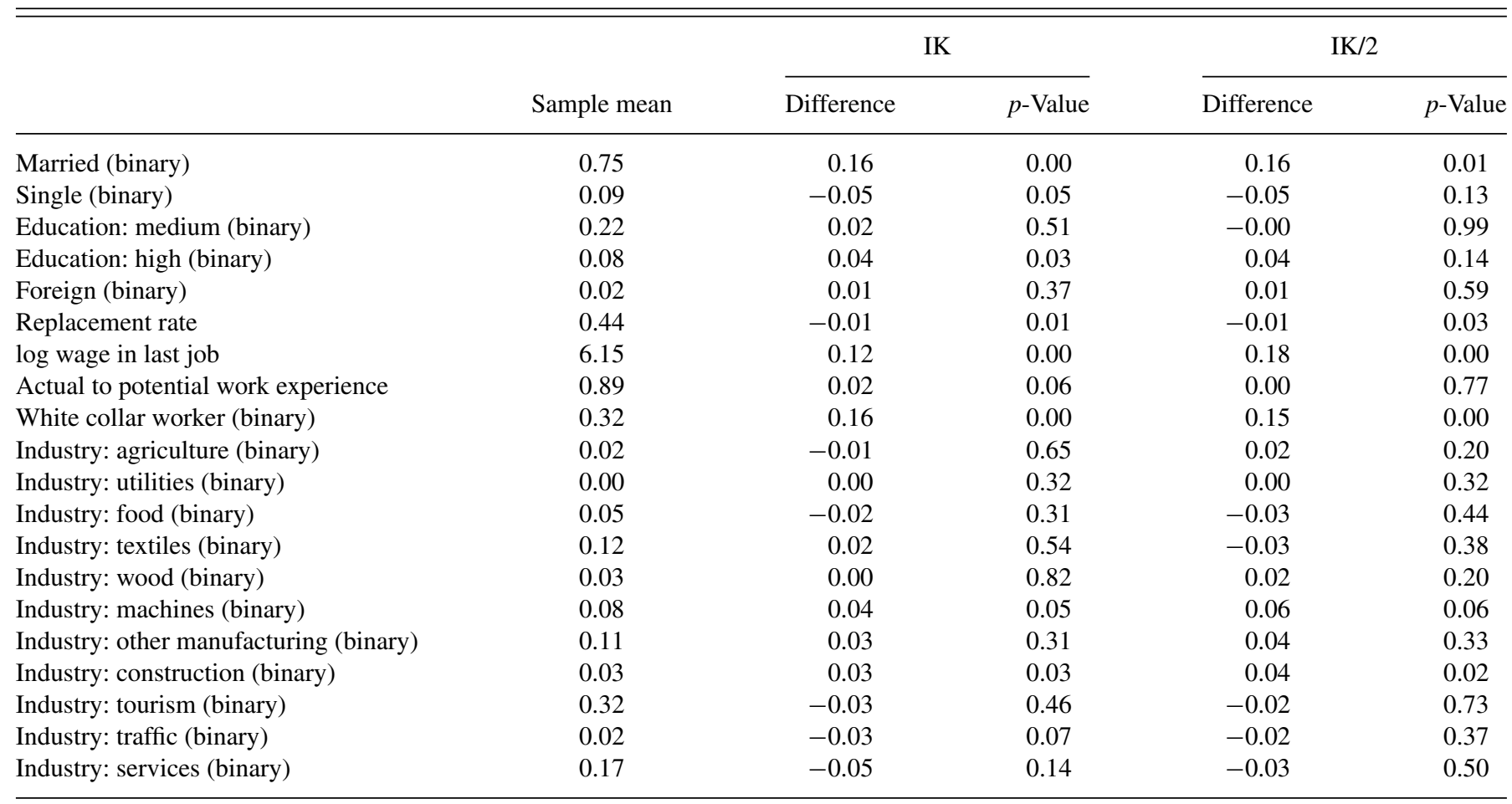

NOTE: "IK," "IK/2" denote the optimal Imbens-Kalyanaraman (2012) bandwidth and half that value in an RDD estimation when using each of the covariates as outcome. $p$-Values are based on analytic standard errors and account for clustering of age (measured in months). 
Table 4. Effect estimates

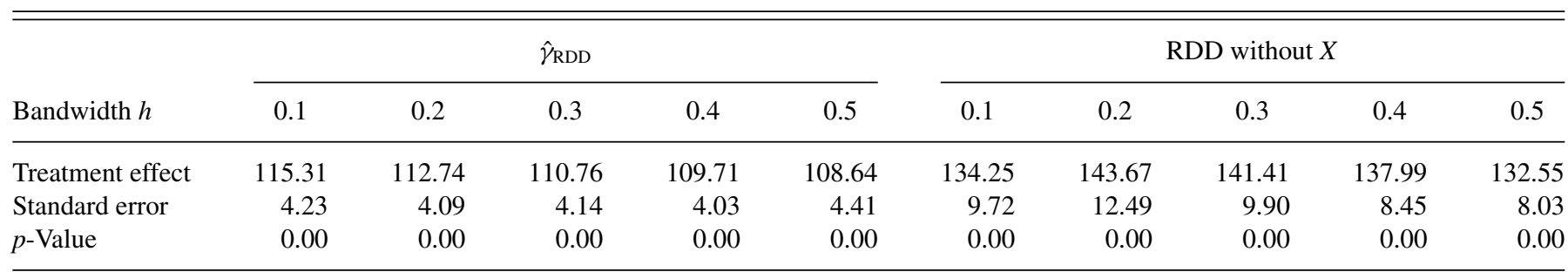

NOTES: The bandwidths $h_{x}, h_{z}$ for the first step estimates of $m^{+}$and $m^{-}$entering $\hat{\gamma}_{\mathrm{RDD}}$ (see Section 3) are picked by least-square cross-validation. For bandwidth $h$ on the running variable $Z$ in $\hat{\gamma}_{\text {RDD }}$ and RDD without $X$, several values are considered as indicated in the table. Standard errors are based on bootstrapping the estimate 999 times. Sample size is 5659 observations. $X$ includes the variables given in Table 3: marital status, education, migration status, replacement rate, log wage in last job, actual to potential work experience, white collar worker, and industry.

threshold, which concerns among others marital status, wage in the last job, and being a white collar worker. (To control the family-wise error rate of multiple testing in Table 3, one may apply the (conservative) Bonferroni correction: divide the nominal level of significance by the number of tested covariates (in our case 20) and reject an individual null hypothesis of covariate balance if the corresponding $p$-value is even lower. For log wage in last job and white collar worker, the null hypothesis is rejected under either bandwidth at the nominal 5\% level of significance.) The results therefore suggest that observations slightly above the age threshold have somewhat more favorable labor market relevant characteristics than those slightly below.

Our RDD estimator derived from Equation (7) controls for differences in $X$ by giving appropriate weights to each of these characteristics, according to their distribution about the threshold. Consider, for example, the variable marital status, which is significantly different in Table 3 . On average, $75 \%$ of the observations in the sample are married, but the (conditional) probability of being married is discontinuous at the threshold: The nonparametric estimates of the probability from the left and right are $63.7 \%$ and $79.9 \%$, respectively. In a symmetric neighborhood about the threshold, the probability of being married is thus $71.8 \%$. Our method proceeds by estimating the outcome unemployment duration for married women left and right of the threshold and multiplying with a weight of 0.718 . An analogous approach applies to unmarried women using a weight of 0.282 . Hence, a weighted average with respect to the fraction of married women in a symmetric neighborhood about the threshold is taken. This removes the discontinuity in marital status: The $63.7 \%$ married women to the left are upweighted with $0.718 / 0.637$, while the $79.9 \%$ married women to the right are down-weighted with $0.718 / 0.799$. Accordingly, the $36.3 \%$ unmarried women to the left are down-weighted with $0.282 / 0.363$, while those $20.1 \%$ to the right are up-weighted with 0.282/0.201. In contrast, RDD estimation not controlling for $X$ compares the unemployment duration left and right of the threshold without weighting, thereby ignoring that there are, for example, fewer married women to the left than to the right of the threshold.

Table 4 presents the results for $\hat{\gamma}_{\text {RDD }}$ when using crossvalidation for the bandwidth selection of $h_{x}, h_{z}$ in the first-step estimation of $\mathrm{m}^{+}$and $\mathrm{m}^{-}$. Different from the simulations in Section 4, however, the covariates now contain both continuous and discrete elements. We therefore apply the method of Racine and Li (2004), which allows for both continuous and discrete regressors by means of product kernels and is implemented in the "np" package of Hayfield and Racine (2008). We use the Epanechnikov, Wang and van Ryzin (1981), and Aitchison and Aitken (1976) kernel functions for continuous, ordered discrete, and unordered discrete covariates, respectively. We consider several choices for bandwidth $h$ in the Epanechnikovbased boundary kernel function for the running variable in (13): $0.1,0.2, \ldots, 0.5$. We also compare the results to RDD regression without covariates based on the "rdd" package with the same bandwidth choice $h$. The standard errors of any method are based on nonparametrically bootstrapping the respective estimates 999 times, that is, randomly resampling the original data with replacement and applying the estimators to the bootstrap samples. The $\hat{\gamma}_{\mathrm{RDD}}$ estimates point to a substantial increase in unemployment duration by about 110 weeks.

The results are highly significant, as the standard errors of roughly 4 weeks are quite moderate. When using RDD without $X$, both the effect of about 140 weeks and the standard error of about 10 weeks are substantially higher. For each bandwidth value considered, the estimates are statistically significantly different between the methods (at the 5\% level based on bootstrapping the differences in the estimates 999 times). This indicates that there might be some confounding due to observed covariates. Also the effects reported in Table 3 columns (3) and (4) of Lalive (2008) when omitting $X$ and either using a global RDD model with a higher order polynomial for the running variable or a local linear model with a very small bandwidth are somewhat higher than $\hat{\gamma}_{\mathrm{RDD}}$ (122 to 126 weeks). In contrast, the effect of 103 weeks presented in column (6) of Table 3 in Lalive (2008) is based on linearly controlling for covariates. Our somewhat higher (and at the 5\% level statistically significantly different) estimates (when bootstrapping the differences) are likely due to using a more flexible specification with respect to the association of $Y$ and $X$.

\section{CONCLUSION}

In this article, the regression discontinuity design (RDD) has been generalized to incorporate covariates $X$ in a fully nonparametric way. Including covariates can reduce the variance and eliminate biases if $X$ is discontinuously distributed at the threshold. It has been shown that the curse of dimensionality does not apply and that the average treatment effect (on the local compliers) can be estimated at rate $n^{-\frac{2}{5}}$ irrespective of the dimension of $X$. For achieving this rate, a boundary RDD estimator has been suggested. We investigated the finite sample properties of our 
estimator in simulations and applied it to estimate the effect of age-dependent unemployment benefits on unemployment duration in Austrian labor market reform, where manipulation at the threshold is a potential concern.

\section{SUPPLEMENTARY MATERIALS}

The supplementary appendix contains all proofs.

\section{ACKNOWLEDGMENTS}

This is a substantially revised version of the 2007 IZA Working Paper 3024. The authors have benefitted from comments by three anonymous referees, the associate editor, and the editor.

\section{FUNDING}

Markus Frölich acknowledges financial support from the Research Cente SFB 884 'Political Economy of Reforms' Project B5, funded by the German Research Foundation (DFG).

[Received January 2015. Revised November 2017.]

\section{REFERENCES}

Aitchison, J., and Aitken, C. (1976), "Multivariate Binary Discrimination by the Kernel Method," Biometrika, 63, 413-420. [747]

Battistin, E., and Rettore, E. (2008), "Ineligibles and Eligible Non-Participants as a Double Comparison Group in Regression-Discontinuity Designs," Journal of Econometrics, 142, 715-730. [737]

Black, D., Galdo, J., and Smith, J. (2007), "Evaluating the Regression Discontinuity Design Using Experimental Data," Mimeo, University of Michigan, USA. [737]

Calonico, S., Cattaneo, M. D., Farrell, M. H., and Titiunik, R. (2018), "Regression Discontinuity Designs Using Covariates," Review of Economics and Statistics, forthcoming. [737]

Calonico, S., Cattaneo, M. D., and Titiunik, R. (2014), "Robust Nonparametric Confidence Intervals for Regression-Discontinuity Designs," Econometrica, 82, 2295-2326. [736]

(2015), "rdrobust: An R Package for Robust Nonparametric Inference in Regression-Discontinuity Designs," $R$ Journal, 7, 38-51. [745]

de Chaisemartin, C. (2017), "Tolerating Defiance? Local Average Treatment Effects Without Monotonicity," Quantitative Economics, 8, 367-396. [739]

Dimmery, D. (2016), Package 'rdd', Manual for the Statistical Software ' $R$ ',' The Comprehensive R Archive Network, available at https://cran.rproject.org/web/packages/rdd/rdd.pdf [744,745]

Dong, Y. (2014), "An Alternative Assumption to Identify LATE in Regression Discontinuity Designs," unpublished manuscript, University of California Irvine. [736,737]

Eugster, B., Lalive, R., Steinhauer, A., and Zweimüller, J. (2017), "Culture, Work Attitudes and Job Search: Evidence From the Swiss Language Border," Journal of the European Economic Association, 15, 1056-1100. [737]

Fisher, R. (1935), Design of Experiments, Edinburgh: Oliver and Boyd. [737]

Frandsen, B., Frölich, M., and Melly, B. (2012), "Quantile Treatment Effects in the Regression Discontinuity Design," Journal of Econometrics, 168, 382-395. [739]

Frölich, M. (2007), "Nonparametric IV Estimation of Local Average Treatment Effects With Covariates," Journal of Econometrics, 139, 35-75. [738]

Frölich, M., and Melly, B. (2013), "Unconditional Quantile Treatment Effects Under Endogeneity," Journal of Business and Economic Statistics (JBES), 31, 346-357. [739]
Gasser, T., and Müller, H. (1979), "Kernel Estimation of Regression Functions," in Smoothing Techniques for Curve Estimation, Lecture Notes in Mathematics 757, eds. T. Gasser, and M. Rosenblatt, Berlin: Springer, pp. 23-68. [740]

Gasser, T., Müller, H., and Mammitzsch, V. (1985), "Kernels for Nonparametric Curve Estimation," Journal of the Royal Statistical Society, Series B, 47, 238-252. [740]

Gelman, A., and Imbens, G. (2018), "Why High-Order Polynomials Should Not be Used in Regression Discontinuity Designs," Business \& Economic Statistics, forthcoming. [736]

Hahn, J., Todd, P., and van der Klaauw, W. (2001), "Identification and Estimation of Treatment Effects with a Regression-Discontinuity Design," Econometrica, 69, 201-209. [736,737,740]

Hayfield, T., and Racine, J. (2008), "Nonparametric Econometrics: The np Package," Journal of Statistical Software, 27, 1-32. [744,747]

Imbens, G. (2001), "Some Remarks on Instrumental Variables," in Econometric Evaluation of Labour Market Policies, eds. M. Lechner, and F. Pfeiffer, Heidelberg: Physica/Springer, pp. 17-42. [739]

Imbens, G., and Angrist, J. (1994), "Identification and Estimation of Local Average Treatment Effects," Econometrica, 62, 467-475. [738]

Imbens, G., and Kalyanaraman, K. (2012), "Optimal Bandwidth Choice for the Regression Discontinuity Estimator," The Review of Economic Studies, 79, 933-959. [736,744]

Imbens, G. W., and Lemieux, T. (2008), "Regression Discontinuity Designs: A Guide to Practice," Journal of Econometrics, 142, 615-635. [736]

Jones, M. (1993), "Simple Boundary Correction for Kernel Density Estimation," Statistics and Computing, 3, 135-146. [740]

Jones, M., and Foster, P. (1996), "A Simple Nonnegative Boundary Correction Method for Kernel Density Estimation," Statistica Sinica, 6, 1005-1013. [740]

Lalive, R. (2008), "How Do Extended Benefits Affect Unemployment Duration? A Regression Discontinuity Approach," Journal of Econometrics, 142, 785-806. [737,746,747]

Lee, D. (2008), "Randomized Experiments From Non-Random Selection in U.S. House Elections," Journal of Econometrics, 142, 675-697. [737]

Lee, D., and Card, D. (2008), "Regression Discontinuity Inference With Specification Error," Journal of Econometrics, 142, 655-674. [736]

Lee, D., and Lemieux, T. (2010), "Regression Discontinuity Designs in Economics," Journal of Economic Literature, 48, 281-355. [736]

Ludwig, J., and Miller, D. L. (2007), "Does Head Start Improve Children's Life Chances? Evidence from a Regression Discontinuity Design," The Quarterly Journal of Economics, 122, 159-208. [745]

McCrary, J. (2008), "Manipulation of the Running Variable in the Regression Discontinuity Design: A Density Test," Journal of Econometrics, 142, 698-714. [736]

Müller, H. (1991), "Smooth Optimum Kernel Estimators Near Endpoints," Biometrika, 78, 521-530. [740]

Newey, W. (1994), "Kernel Estimation of Partial Means and a General Variance Estimator," Econometric Theory, 10, 233-253. [739]

Neyman, J. (1923), "On the Application of Probability Theory to Agricultural Experiments. Essay on Principles," Statistical Science, 5, 463-480. [737]

Porter, J. (2003), "Estimation in the Regression Discontinuity Model," Mimeo. [736]

Racine, J., and Li, Q. (2004), "Nonparametric Estimation of Regression Functions with Both Categorical and Continuous Data," Journal of Econometrics, 119, 99-130. [740,747]

Rubin, D. (1978), "Bayesian Inference for Causal Effects: The Role of Randomization," Annals of Statistics, 6, 34-58. [737]

Tenreiro, C. (2013), "Boundary Kernels for Distribution Function Estimation," REVSTAT-Statistical Journal, 11, 169-190. [740]

Trochim, W. (1984), Research Design for Program Evaluation: The RegressionDiscontinuity Approach, Beverly Hills: Sage Publications. [737]

Urquiola, M., and Verhoogen, E. (2009), "Class-Size Caps, Sorting, and the Regression-Discontinuity Design," The American Economic Review, 99, 179-215. [737,738]

van der Klaauw, W. (2008), "Breaking the Link Between Poverty and Low Student Achievement: An Evaluation of Title I," Journal of Econometrics, 142, 731-756. [736,737]

Wang, M., and van Ryzin, J. (1981), "A Class of Smooth Estimators for Discrete Distributions," Biometrika, 68, 301-309. [747] 